\title{
Wave Polarization Analyzed by Singular Value Decomposition of the Spectral Matrix in the Presence of Noise
}

\author{
Ulrich Taubenschuss $^{1}$ (D) Ondřej Santolík ${ }^{1,2}$ (D)
}

Received: 13 May 2018 / Accepted: 9 August 2018 / Published online: 19 August 2018

(c) The Author(s) 2018

\begin{abstract}
Analysis of wave polarization provides wave propagation parameters and enables an identification of modes in space plasmas. It is based on measurements of several components of fluctuating electromagnetic fields. This technique has become a conventional part of modern instrumentation onboard scientific spacecraft. A definition of the degree of polarization can be reduced to a very basic form, i.e., the ratio of a signal's polarized power to its total power. However, this simple definition can have several different realizations which depend mainly on the underlying assumptions about separating the polarized (coherent) part from the unpolarized part (noise). After reviewing polarization of a plane wave in two and three dimensions, we examine the singular value decomposition technique for a complex spectral matrix as well as for a real spectral matrix. The meaning of singular values is explained, and we show to what extent the singular values are able to contribute to a separation between polarized signal and noise. Finally, our theoretical findings are verified with synthetic data as well as with whistler-mode chorus wave observations from the THEMIS spacecraft.
\end{abstract}

Keywords Polarization analysis $\cdot$ Plane wave $\cdot$ Hermitian spectral matrix $\cdot$ Singular value decomposition - Spectral averaging · Singular values · Eigenvalues · Stokes parameters · Degree of polarization $\cdot$ Ellipticity $\cdot$ Planarity $\cdot$ Wave normal vector

\section{Introduction}

Polarization is a fundamental feature of waves in plasmas. With regard to space plasmas, information about the polarization enables, together with a wave's frequency, an identification of the mode of propagation. A magnetospheric plasma can host a variety of electromagnetic wave modes like whistler-mode hiss (Li et al. 2015), chorus (Santolík 2008), lightning whistlers (Storey 1953), electromagnetic ion cyclotron waves (Meredith et al. 2003), or the prominent auroral kilometric radiation (AKR) in the RX and LO-mode (Gurnett 1974; Wu and Lee

Ulrich Taubenschuss

ut@ufa.cas.cz

1 Institute of Atmospheric Physics, Czech Academy of Sciences, Bočni II/1401, 14131 Prague, Czech Republic

2 Faculty of Mathematics and Physics, Charles University, V Holešovičkách 2, 18000 Prague,

Czech Republic 
1979), just to name a few. The latter dominates the higher frequency part of the radio spectrum not only at the Earth but also at the gas giant planets Jupiter (KOM/HOM/DAM), Saturn (SKR), Uranus (UKR) and Neptune (NKR) (Zarka 1998; Fischer et al. 2009; Kurth et al. 2017). Each naturally produced radio emission has its own distinct polarization characteristics which depend on the generation mechanism as well as on the composition of the propagation medium. Thus, for a deeper understanding of these waves, it is essential to thoroughly investigate their polarization.

Modern spacecraft instrumentation often provides us with multicomponent measurements of electric and magnetic fields which can serve as essential input data for polarization analysis. Exemplary missions/instrumentations are: Polar/PWI (Gurnett et al. 1995), Cassini/RPWS (Gurnett et al. 2004), DEMETER/IMSC/ICE (Parrot et al. 2006; Berthelier et al. 2006), Cluster/STAFF (Cornilleau-Wehrlin et al. 1997), THEMIS/SCM/EFI (Le Contel et al. 2008; Bonnell et al. 2008), Van Allen Probes/EMFISIS (Kletzing et al. 2013) and MMS/FIELDS (Torbert et al. 2016). Relevant missions in preparation are Taranis/IME-BF, Solar Orbiter/RPW and JUICE/RPWI.

Multicomponent measurements of the wave's magnetic field (B) oscillations along three independent directions can be summarized in a $3 \times 3$ complex spectral matrix $\mathbf{J}$ of the form

$$
\mathbf{J}=\left(\begin{array}{ccc}
\left\langle B_{x} B_{x}^{*}\right\rangle & \left\langle B_{x} B_{y}^{*}\right\rangle & \left\langle B_{x} B_{z}^{*}\right\rangle \\
\left\langle B_{y} B_{x}^{*}\right\rangle & \left\langle B_{y} B_{y}^{*}\right\rangle & \left\langle B_{y} B_{z}^{*}\right\rangle \\
\left\langle B_{z} B_{x}^{*}\right\rangle & \left\langle B_{z} B_{y}^{*}\right\rangle & \left\langle B_{z} B_{z}^{*}\right\rangle
\end{array}\right)=\left(\begin{array}{ccc}
J_{x x} & J_{x y} & J_{x z} \\
J_{x y}^{*} & J_{y y} & J_{y z} \\
J_{x z}^{*} & J_{y z}^{*} & J_{z z}
\end{array}\right) .
$$

A similar matrix can be established for the wave's electric field $\mathbf{E}$ as well. The operator $\langle\ldots\rangle$ indicates that averaging of the signal has to be performed. $\mathbf{B}$ as a complex quantity can be generated from the real measured signal in the time domain by means of the Fourier transform, thereby encoding amplitude and starting phase angle of the harmonic oscillation in the real and imaginary part. Matrix elements representing auto-spectral power are positioned along the main diagonal of $\mathbf{J}$, and cross-spectral powers form the off-diagonal elements. One important property of the spectral matrix is that it is a Hermitian matrix, i.e., it equals its transpose and complex conjugate. (Complex conjugate is indicated by a *-symbol.) Furthermore, it is important to note that the components of $\mathbf{B}$, or $\mathbf{E}$, are assumed to be given in an orthogonal coordinate system by means of a proper antenna calibration.

An electromagnetic wave can be treated locally as a plane wave if observed in the far field of the source with an antenna system of sufficiently small aperture. Then, the curvature of the wavefront, i.e., the curvature of the surface of constant phase, is negligible. If the $z$-axis of the coordinate system is chosen such that it points perpendicularly to the plane of the wavefront, then $B_{z}=0$, and all elements in $\mathbf{J}$ which include $B_{z}$ vanish. This coordinate frame shall be called the wave-frame. For the magnetic field of a harmonic plane wave, the $z$-axis of the wave-frame coincides with the direction of the wave vector $\mathbf{k}$, since a linearized version of $\nabla \cdot \mathbf{B}=0$ ensures that $\mathbf{B} \perp \mathbf{k}$.

As mentioned above, the spectral matrix is Hermitian and positive semi-definite, with a symmetric real part and an anti-symmetric imaginary part. For a fully polarized plane wave expressed in its wave-frame, a separation into real and imaginary part looks as follows:

$$
\mathbf{J}_{\mathrm{p}}=\left(\begin{array}{ccc}
J_{\mathrm{p}, x x} & \mathfrak{R}\left\{J_{\mathrm{p}, x y}\right\} & 0 \\
\mathfrak{R}\left\{J_{\mathrm{p}, x y}\right\} & J_{\mathrm{p}, y y} & 0 \\
0 & 0 & 0
\end{array}\right)+i\left(\begin{array}{ccc}
0 & \mathfrak{J}\left\{J_{\mathrm{p}, x y}\right\} & 0 \\
-\mathfrak{I}\left\{J_{\mathrm{p}, x y}\right\} & 0 & 0 \\
0 & 0 & 0
\end{array}\right) .
$$

Subscript $p$ shall indicate that $\mathbf{J}_{\mathrm{p}}$ belongs to a $100 \%$ polarized wave. Symbols $\mathfrak{R}\{\}$ and $\mathfrak{I}\{\}$ specify real and imaginary part of a complex quantity, respectively. 
A wave which is not fully polarized is said to be partially polarized, meaning that it also contains an unpolarized component. Moreover, unpolarized radiation is introduced by other natural or instrumental sources of noise, although instrumental noise is usually dominant. Thus, the complete spectral matrix for noise $\mathbf{J}_{\mathrm{n}}$ (subscript $\mathrm{n}$ stands for noise) can be modeled in the wave-frame as

$$
\mathbf{J}_{\mathrm{n}}=\mathbf{J}_{\mathrm{n} 1}+\mathbf{J}_{\mathrm{n} 2}=\left(\begin{array}{ccc}
\tilde{a} & 0 & 0 \\
0 & \tilde{a} & 0 \\
0 & 0 & 0
\end{array}\right)+\left(\begin{array}{ccc}
c & 0 & 0 \\
0 & c & 0 \\
0 & 0 & c
\end{array}\right)=\left(\begin{array}{ccc}
a & 0 & 0 \\
0 & a & 0 \\
0 & 0 & c
\end{array}\right),
$$

with $a=\tilde{a}+c . \mathbf{J}_{\mathrm{n} 1}$ is the modeled spectral matrix for the noise component of the partially polarized plane wave. $\mathbf{J}_{\mathrm{n} 2}$ is the spectral matrix for the isotropic instrumental/background noise. Both matrices are entirely real, and, if expressed in the wave-frame, all cross-spectral powers are zero. Thus, all coherencies $\left|J_{i j}\right| / \sqrt{J_{i i} J_{j j}}$ are zero. The auto-spectral powers $\tilde{a}$ and $c$ represent noise powers along the three orthogonal directions. They are proportional to variances of noise amplitudes if modeled from a random Gaussian distribution.

$\mathbf{J}_{\mathrm{n} 2}$ is independent of the orientation of the coordinate system, i.e., $\mathbf{J}_{\mathrm{n} 2}$ is invariant under the unitary transformation. On the other hand, $\mathbf{J}_{\mathrm{n} 1}$ remains incoherent only if expressed in the wave-frame. Any rotation of the coordinate system about a nonzero polar angle will generate nonzero off-diagonal elements. Nevertheless, we keep on using the term "noise" even if $\mathbf{J}_{\mathrm{n} 1}$ is not fully incoherent from a three-dimensional point of view. It will be shown later how this special approach will help to understand results from singular value decomposition of the spectral matrix.

We also want to stress that $\mathbf{J}_{\mathrm{n}}$ from Eq. (3) is a highly idealized case used for modeling. In reality, off-diagonal elements of $\mathbf{J}_{\mathrm{n}}$ will almost never be exactly zero because of experimental inaccuracies of the spectral analysis.

As already pointed out by Stokes (1852), every beam of light can be considered as a linear superposition of fully polarized and unpolarized light. Thus, it makes sense to define the degree of polarization as the ratio of power contained in the polarized part to the total power. The total power is equal to the trace of the corresponding spectral matrix. So, what we need to do is to split the measured $\mathbf{J}$ into a sum of $\mathbf{J}_{\mathrm{p}}$, the fully polarized component, and $\mathbf{J}_{\mathbf{n}}$, the totally unpolarized component, according to

$$
\mathbf{J}=\mathbf{J}_{\mathrm{p}}+\mathbf{J}_{\mathrm{n}}
$$

Subsequently, the degree of polarization $D_{\mathrm{p}}$ can be defined as

$$
D_{\mathrm{p}}=\frac{\operatorname{tr}\left(\mathbf{J}_{\mathrm{p}}\right)}{\operatorname{tr}(\mathbf{J})} \text {. }
$$

The operator tr indicates the trace of a matrix. A value of $D_{\mathrm{p}}=1$ characterizes a fully polarized signal, $0<D_{\mathrm{p}}<1$ means partially polarized, and $D_{\mathrm{p}}=0$ results from totally unpolarized radiation.

\subsection{A Brief Summary on Polarization of a Plane Wave}

As can be seen from expressions (2) and (3), $\mathbf{J}=\mathbf{J}_{\mathrm{p}}+\mathbf{J}_{\mathrm{n}}$ can be reduced to a two-dimensional (2D) problem in the wave-frame of a plane wave when neglecting contributions from noise along the $z$-direction $\left(J_{z z}=0\right)$. The spectral matrix becomes 


$$
\mathbf{J}_{2}=\left(\begin{array}{cc}
\left\langle B_{x} B_{x}^{*}\right\rangle & \left\langle B_{x} B_{y}^{*}\right\rangle \\
\left\langle B_{y} B_{x}^{*}\right\rangle & \left\langle B_{y} B_{y}^{*}\right\rangle
\end{array}\right)=\left(\begin{array}{cc}
J_{x x} & J_{x y} \\
J_{x y}^{*} & J_{y y}
\end{array}\right) .
$$

Subscript 2 shall mark the 2D version of $\mathbf{J}$. The oscillations of $B_{x}$ and $B_{y}$ describe a rotating vector which rotates with a certain frequency (monochromatic wave). For a fully polarized wave, the phase shift between $B_{x}$ and $B_{y}$ is constant. Any random jumps in phase are attributed to noise. In the most general case, the tip of vector $\mathbf{B}$ delineates an ellipse, referring to elliptical polarization. Two special states are circular polarization (tip of $\mathbf{B}$ follows a circle) and linear polarization (ellipse is compressed to a line).

Polarization properties are often expressed in terms of the Stokes parameters (see, e.g., Born and Wolf 1999; Thompson et al. 2017). In the wave-frame, the 2D Stokes parameters are

$$
\begin{gathered}
S=J_{x x}+J_{y y}=\left\langle B_{x} B_{x}^{*}\right\rangle+\left\langle B_{y} B_{y}^{*}\right\rangle, \\
Q=J_{x x}-J_{y y}=\left\langle B_{x} B_{x}^{*}\right\rangle-\left\langle B_{y} B_{y}^{*}\right\rangle, \\
U=J_{x y}+J_{x y}^{*}=2 \mathfrak{R}\left\{J_{x y}\right\}=2 \mathfrak{R}\left\{\left\langle B_{x} B_{y}^{*}\right\rangle\right\}, \\
V=i\left(J_{x y}^{*}-J_{x y}\right)=2 \mathfrak{S}\left\{J_{x y}\right\}=2 \mathfrak{\Im}\left\{\left\langle B_{x} B_{y}^{*}\right\rangle\right\} .
\end{gathered}
$$

Sometimes, $Q, U$ and $V$ are normalized, i.e., divided by $S$. The Stokes parameter $S$ equals the total power of the signal, which is the sum of its polarized part and noise. The latter contributes only to the real part of $\mathbf{J}$ since any imaginary part nonequal to zero already indicates a polarized component. Moreover, matrix elements from noise appear only along the main diagonal of $\mathbf{J}$. Then, $U$ and $V$, which are composed from off-diagonal elements of $\mathbf{J}$, are noise-free. Furthermore, the noise power in $x$-direction equals the noise power in the $y$-direction. Thus, noise cancels out in $Q$ as well. Under such circumstances, the quantity $\left(Q^{2}+U^{2}+V^{2}\right)^{1 / 2}$ can be considered as the power of the fully polarized part only.

The Stokes parameters are related to the shape and orientation of the polarization ellipse in the $[x, y]$-plane of the wave-frame. The following relations hold:

$$
\begin{gathered}
\tau=\frac{1}{2} \arctan (U / Q), \\
\beta=\frac{1}{2} \arcsin \left(V /\left[Q^{2}+U^{2}+V^{2}\right]^{1 / 2}\right) .
\end{gathered}
$$

The angle $\tau$ describes the tilt between the semi-major axis of the polarization ellipse and the $x$-axis of the wave-frame. The quantity

$$
\varepsilon=\tan (\beta)
$$

is called ellipticity and equals the ratio of semi-minor axis to semi-major axis of the polarization ellipse. It should be noted that $\tau$ and $\varepsilon$ are computed using only Stokes parameters from the fully polarized part of the wave. $\varepsilon=0$ means linear polarization, $\varepsilon=-1$ is left-handed circular polarization, and $\varepsilon=+1$ is right-handed circular polarization. Everything between 0 and 1 (or -1 ) is referred to as elliptical polarization. The terms left-handed $(\varepsilon<0)$ and right-handed $(\varepsilon>0)$ can be based on the IEEE 1997 standard (IEEE 1998) and describe polarization relative to the direction of the wave vector $\mathbf{k}$ ( $\mathbf{k}$ points along $+z$ of the wave-frame). If an observer is located at positive $z$ and looks down on the plane of measurement ( $[x, y]$-plane), then he sees the wave approaching from below (along $+z$-direction). Successively measured B vectors of the 
approaching wave in the $[x, y]$-plane seem to rotate around the $z$-axis. If the sense of circular polarization of the wave is left-handed, then $\mathbf{B}$ rotates clockwise. On the other hand, for right-handed polarization, $\mathbf{B}$ rotates counterclockwise in the plane of measurement for an observer looking into the approaching wave.

However, the sense of polarization in plasma physics is defined with respect to the direction of the ambient magnetic field $\mathbf{B}_{\mathbf{0}}$ instead of $\mathbf{k}$. Then, the phrase "right-handed" corresponds to the same sense of rotation in which electrons are gyrating around magnetic field lines: clockwise, if looking along $\mathbf{B}_{\mathbf{0}}$, or counterclockwise if looking against $\mathbf{B}_{\mathbf{0}}$ (opposite for left-handed).

The following $2 \mathrm{D}$ degrees of polarization can be specified with the Stokes parameters:

$$
\begin{aligned}
& D_{\mathrm{p} 2}=\left[Q^{2}+U^{2}+V^{2}\right]^{\frac{1}{2}} / S \quad \ldots 2 \text { D degree of (total) polarization, } \\
& D_{l 2}=\left[Q^{2}+U^{2}\right]^{\frac{1}{2}} / S \quad \ldots 2 \text { D degree of linear polarization, } \\
& D_{c 2}=V / S \quad \ldots 2 \text { D degree of circular polarization. }
\end{aligned}
$$

$D_{\mathrm{p} 2}$ and $D_{l 2}$ range from 0 to 1 , whereas $D_{c 2}$ is defined between $-1(\mathrm{LH})$ and $+1(\mathrm{RH})$.

The 2D degree of total polarization, or simply degree of polarization, quantifies the ratio of power in the wave's polarized part to the total power (see also Eq. (5)). It can be reformulated by inserting the expressions for $S, Q, U$ and $V$ from Eqs. (7)-(10) into Eq. (14). This yields (see Appendix A)

$$
\begin{aligned}
D_{\mathrm{p} 2} & =\left[\frac{\left(J_{x x}-J_{y y}\right)^{2}+\left(J_{x y}+J_{x y}^{*}\right)^{2}-\left(J_{x y}^{*}-J_{x y}\right)^{2}}{\left(J_{x x}+J_{y y}\right)^{2}}\right]^{\frac{1}{2}} \\
& \vdots \\
& =\left[1-\frac{4 \operatorname{det}\left(\mathbf{J}_{2}\right)}{\operatorname{tr}^{2}\left(\mathbf{J}_{2}\right)}\right]^{\frac{1}{2}} \\
= & {\left[\frac{2 \operatorname{tr}\left(\mathbf{J}_{2}^{2}\right)}{\operatorname{tr}^{2}\left(\mathbf{J}_{2}\right)}-1\right]^{\frac{1}{2}} . }
\end{aligned}
$$

The operator det means the determinant, and tr stands for trace. $\mathbf{J}^{2}$ refers to matrix multiplication, i.e., $\mathbf{J}^{2}=\mathbf{J} \cdot \mathbf{J}$. The determinant and the trace are scalar invariants of the spectral matrix. These quantities do not change under unitary transformation (rotation of a matrix) into a different coordinate system, i.e., $\mathbf{J}$ and $\mathbf{J}^{\prime}$, with

$$
\mathbf{J}^{\prime}=\mathbf{R} \cdot \mathbf{J} \cdot \mathbf{R}^{-1}=\mathbf{R} \cdot \mathbf{J} \cdot \mathbf{R}^{T},
$$

have the same invariants. $\mathbf{R}$ is the orthonormal rotation matrix. Thus, the value of $D_{\mathrm{p} 2}$ is independent of the coordinate system in which $\mathbf{J}$ is expressed.

The invariants of $\mathbf{J}$ used in Eq. (18) can be replaced by combinations of the two eigenvalues $\lambda_{0}$ and $\lambda_{1}\left(\lambda_{1} \geq \lambda_{0} \geq 0\right)$ of $\mathbf{J}$, if $\mathbf{J}$ is expanded on an orthonormal basis of eigenvectors (2D problem). A solution of the corresponding eigenvalue problem is presented in Sect. 2.1. 
Besides definitions (14) and (18), a third expression for the 2D degree of polarization is (Samson 1973)

$$
D_{\mathrm{p} 2}=\frac{\lambda_{1}-\lambda_{0}}{\lambda_{0}+\lambda_{1}}
$$

Now, the 2D spectral matrix from Eq. (6) shall be expressed in terms of the 2D Stokes parameters. One gets

$$
\mathbf{J}_{2}=\frac{1}{2}\left(\begin{array}{cc}
S+Q & U+i V \\
U-i V & S-Q
\end{array}\right)
$$

Furthermore, $\mathbf{J}_{2}$ can be split into a sum involving the identity matrix $\boldsymbol{\sigma}_{S}$ and the three Pauli spin matrices $\sigma_{Q}, \sigma_{U}$ and $\sigma_{V}$ (generators of the $\mathrm{SU}(2)$ symmetry group) (Wiener 1930; Fano 1957; Samson 1973; Setälä et al. 2002), yielding

$$
\mathbf{J}_{2}=\frac{1}{2}\left(S \boldsymbol{\sigma}_{S}+Q \boldsymbol{\sigma}_{Q}+U \boldsymbol{\sigma}_{U}+V \boldsymbol{\sigma}_{V}\right)
$$

with

$$
\sigma_{S}=\left(\begin{array}{ll}
1 & 0 \\
0 & 1
\end{array}\right), \quad \sigma_{Q}=\left(\begin{array}{cc}
1 & 0 \\
0 & -1
\end{array}\right), \quad \sigma_{U}=\left(\begin{array}{ll}
0 & 1 \\
1 & 0
\end{array}\right), \quad \sigma_{V}=\left(\begin{array}{cc}
0 & i \\
-i & 0
\end{array}\right) .
$$

Note that our $\sigma_{V}$ is $(-1)$ times the usual Pauli matrix in order to be compatible with $J_{x y}=\left\langle B_{x} B_{y}^{*}\right\rangle$ and the convention $V>0$ for a right-handed sense of polarization.

A similar strategy for more than two dimensions has been pursued by Samson (1973) and Barakat (1977) in the fields of geophysics and optics, respectively. In the three-dimensional case, the 2D Pauli spin matrices are replaced by the set of eight Gell-Mann matrices, or generators of the SU(3) symmetry group (Gell-Mann 1962). These $3 \times 3$ matrices are, like the 2D Pauli spin matrices, linearly independent, traceless and Hermitian. Furthermore, they are trace orthogonal $(\operatorname{tr}(\mathbf{A} \cdot \mathbf{B})=0)$ with each other. Again, the set of generators is complemented by the $3 \times 3$ identity matrix, resulting in a decomposition of $\mathbf{J}$ into nine components. In three dimensions, one gets (Setälä et al. 2002; Sheppard 2011)

$$
\begin{aligned}
& \mathbf{J}=\frac{\sigma_{0}}{3}\left(\begin{array}{lll}
1 & 0 & 0 \\
0 & 1 & 0 \\
0 & 0 & 1
\end{array}\right)+\frac{\sigma_{1}}{3}\left(\begin{array}{ccc}
0 & 1 & 0 \\
1 & 0 & 0 \\
0 & 0 & 0
\end{array}\right)+\frac{\sigma_{2}}{3}\left(\begin{array}{ccc}
0 & -i & 0 \\
i & 0 & 0 \\
0 & 0 & 0
\end{array}\right) \\
& +\frac{\sigma_{3}}{3}\left(\begin{array}{ccc}
1 & 0 & 0 \\
0 & -1 & 0 \\
0 & 0 & 0
\end{array}\right)+\frac{\sigma_{4}}{3}\left(\begin{array}{ccc}
0 & 0 & 1 \\
0 & 0 & 0 \\
1 & 0 & 0
\end{array}\right)+\frac{\sigma_{5}}{3}\left(\begin{array}{ccc}
0 & 0 & -i \\
0 & 0 & 0 \\
i & 0 & 0
\end{array}\right) \\
& +\frac{\sigma_{6}}{3}\left(\begin{array}{ccc}
0 & 0 & 0 \\
0 & 0 & 1 \\
0 & 1 & 0
\end{array}\right)+\frac{\sigma_{7}}{3}\left(\begin{array}{ccc}
0 & 0 & 0 \\
0 & 0 & -i \\
0 & i & 0
\end{array}\right) \\
& +\frac{\sigma_{8}}{3}\left(\begin{array}{ccc}
1 / \sqrt{3} & 0 & 0 \\
0 & 1 / \sqrt{3} & 0 \\
0 & 0 & -2 / \sqrt{3}
\end{array}\right) \text {. }
\end{aligned}
$$


The 9 coefficients $\sigma_{0}-\sigma_{8}$ are, by analogy to the 2D problem, interpreted as the nine 3D Stokes parameters. It should be noted that different versions of the Gell-Mann matrices exist in the literature, e.g., in Samson (1973), leading to slightly different expressions for the coefficients. The $\sigma$ are all real quantities, and their explicit forms for our purpose are

$$
\begin{aligned}
& \sigma_{0}=J_{x x}+J_{y y}+J_{z z}, \\
& \sigma_{1}=\frac{3}{2}\left(J_{x y}+J_{x y}^{*}\right), \quad \sigma_{2}=\frac{3}{2} i\left(J_{x y}-J_{x y}^{*}\right), \quad \sigma_{3}=\frac{3}{2}\left(J_{x x}-J_{y y}\right), \\
& \sigma_{4}=\frac{3}{2}\left(J_{x z}+J_{x z}^{*}\right), \quad \sigma_{5}=\frac{3}{2} i\left(J_{x z}-J_{x z}^{*}\right), \\
& \sigma_{6}=\frac{3}{2}\left(J_{y z}+J_{y z}^{*}\right), \quad \sigma_{7}=\frac{3}{2} i\left(J_{y z}-J_{y z}^{*}\right), \\
& \sigma_{8}=\frac{\sqrt{3}}{2}\left(J_{x x}+J_{y y}-2 J_{z z}\right) .
\end{aligned}
$$

The parameter $\sigma_{0}$ is the total power in three dimensions, similar to $S$ from the 2D case. $\sigma_{3}$ represents $Q$ in the $[x, y]$-plane. $\sigma_{1}, \sigma_{4}$ and $\sigma_{6}$ are like $U$ in the $[x, y]-,[x, z]$ - and $[y, z]$-plane, respectively. $\sigma_{2}, \sigma_{5}$ and $\sigma_{7}$ are like $V$ in the $[x, y]-,[x, z]-$ and $[y, z]$-plane, respectively. The parameter $\sigma_{8}$ is a linear combination of $Q$ in the $[x, z]$-plane and $Q$ in the $[y, z]$-plane $\left(\sigma_{8}=\sqrt{3} / 2\left[\left(J_{x x}-J_{z z}\right)+\left(J_{y y}-J_{z z}\right)\right]\right)$.

With the 3D Stokes parameters, the spectral matrix takes the form

$$
\mathbf{J}=\left(\begin{array}{ccc}
J_{x x} & J_{x y} & J_{x z} \\
J_{x y}^{*} & J_{y y} & J_{y z} \\
J_{x z}^{*} & J_{y z}^{*} & J_{z z}
\end{array}\right)=\frac{1}{3}\left(\begin{array}{ccc}
\sigma_{0}+\sigma_{3}+\frac{1}{\sqrt{3}} \sigma_{8} & \sigma_{1}-i \sigma_{2} & \sigma_{4}-i \sigma_{5} \\
\sigma_{1}+i \sigma_{2} & \sigma_{0}-\sigma_{3}+\frac{1}{\sqrt{3}} \sigma_{8} & \sigma_{6}-i \sigma_{7} \\
\sigma_{4}+i \sigma_{5} & \sigma_{6}+i \sigma_{7} & \sigma_{0}-\frac{2}{\sqrt{3}} \sigma_{8}
\end{array}\right) .
$$

Following the same pattern as in $2 \mathrm{D}$, a degree of polarization can be formulated in three dimensions as

$$
D_{\mathrm{p} 3}=\frac{1}{\sqrt{3}} \frac{\left(\sigma_{1}^{2}+\sigma_{2}^{2}+\cdots+\sigma_{8}^{2}\right)^{\frac{1}{2}}}{\sigma_{0}} .
$$

The factor $1 / \sqrt{3}$ is necessary to ensure that $D_{\mathrm{p} 3}=1$ for a fully polarized wave (see Appendix B). Inserting $\sigma_{0}$ to $\sigma_{8}$ into Eq. (27) yields another form of this expression:

$$
D_{\mathrm{p} 3}=\left[\frac{3}{2} \frac{\operatorname{tr}\left(\mathbf{J}^{2}\right)}{\operatorname{tr}^{2}(\mathbf{J})}-\frac{1}{2}\right]^{\frac{1}{2}} .
$$

Furthermore, the terms $\operatorname{tr}\left(\mathbf{J}^{2}\right)$ and $\operatorname{tr}^{2}(\mathbf{J})$ can be reformulated using the eigenvalues $\lambda_{i}$ ( $i=0,1,2)$ of $\mathbf{J}$, leading to

$$
D_{\mathrm{p} 3}=\frac{\left[\lambda_{0}^{2}+\lambda_{1}^{2}+\lambda_{2}^{2}-\lambda_{0} \lambda_{1}-\lambda_{0} \lambda_{2}-\lambda_{1} \lambda_{2}\right]^{\frac{1}{2}}}{\lambda_{0}+\lambda_{1}+\lambda_{2}} .
$$


Equations (27), (28) and (29) are three different ways for computing a 3D degree of polarization, but they all yield identical results. They are just formulated in terms of different parameters: the 3D Stokes parameters, the invariants of the spectral matrix, or the eigenvalues of the spectral matrix. Nevertheless, the validity of this approach for expanding from two to three dimensions using the Gell-Mann matrices may be questioned. An interpretation of the 3D Stokes parameters is difficult, besides that the 3D version is not simply a superposition of three two-dimensional problems (polarization in $[x, y]-,[x, z]-$ and $[y$, $z]$-plane). As shown above, the two Stokes parameters $Q$ from the $[x, z]$ - and $[y, z]$-plane have to be combined to $\sigma_{8}$ in order to enable a full decomposition of $\mathbf{J}$ into trace orthogonal matrices. Another critical point is that the $3 \mathrm{D}$ formulation of the degree of polarization does not reduce to its $2 \mathrm{D}$ version by simply ignoring the $z$-dimension. Finally, as can be seen from Eq. (29), $D_{\mathrm{p} 3}$ becomes zero only if all three eigenvalues are equal. As will be shown later, this implies a $\mathbf{J}_{\mathrm{n}}$ for perfectly isotropic noise.

As mentioned earlier, the Hermitian spectral matrix can be decomposed on the basis of orthonormal eigenvectors. This method was already described by Samson (1973) who called it expansion in non-disjoint idempotent matrices. The topic was later picked up again by Ellis et al. (2005) who re-interpreted the results. Their starting point was a diagonalization of the Hermitian matrix $\mathbf{J}$ of the form

$$
\mathbf{J}=\mathbf{U} \cdot \boldsymbol{\Lambda} \cdot \mathbf{U}^{H}
$$

where matrix $\mathbf{U}$ holds the (complex) eigenvectors along its columns, and real matrix $\boldsymbol{\Lambda}$ is a diagonal matrix holding the eigenvalues along the main diagonal. More details about this diagonalization are provided in Sect. 2. Proper restructuring of Eq. (30) leads to (see Appendix C)

$$
\mathbf{J}=\left(\lambda_{2}-\lambda_{1}\right) \mathbf{U} \cdot\left(\begin{array}{lll}
1 & 0 & 0 \\
0 & 0 & 0 \\
0 & 0 & 0
\end{array}\right) \cdot \mathbf{U}^{H}+\left(\lambda_{1}-\lambda_{0}\right) \mathbf{U} \cdot\left(\begin{array}{lll}
1 & 0 & 0 \\
0 & 1 & 0 \\
0 & 0 & 0
\end{array}\right) \cdot \mathbf{U}^{H}+\lambda_{0} \mathbf{U} \cdot\left(\begin{array}{lll}
1 & 0 & 0 \\
0 & 1 & 0 \\
0 & 0 & 1
\end{array}\right) \cdot \mathbf{U}^{H} .
$$

Now, the argument of Ellis et al. (2005) is the following. The second and third terms on the right-hand side of Eq. (31) are entirely real, meaning that they contain all the unpolarized light. On the other hand, the first term has an imaginary part nonequal to zero, i.e., it corresponds to fully polarized light (Ellis and Dogariu 2005). This way, the polarized part of the signal gets split from the unpolarized part. The three respective power factors are given as functions of the eigenvalues $\left(\lambda_{2}-\lambda_{1}, \lambda_{1}-\lambda_{0}\right.$, and $\left.\lambda_{0}\right)$ in front of each term. They can be used for a 3D degree of polarization (ratio of polarized to total power), yielding

$$
D_{\mathrm{p} 3 e}=\frac{\lambda_{2}-\lambda_{1}}{\lambda_{0}+\lambda_{1}+\lambda_{2}} .
$$

The subscript $p 3 e$ shall refer to Ellis et al. (2005) in order to differentiate it from $D_{\mathrm{p} 3}$ in Eq. (29). In contrast to $D_{\mathrm{p} 3}, D_{\mathrm{p} 3 e}$ reduces to the correct version in 2D by simply skipping the z-dimension (see Eq. (20) for comparison). Nevertheless, it still makes certain assumptions about the structure of the noise spectral matrix as will be shown later on. 


\subsection{Key Features of the Wave Propagation Singular Value Decomposition (SVD) Technique}

Santolík et al. (2003) presented a method for deriving a robust estimate for the direction of the wave vector assuming the presence of a plane wave. This method is known under the name PRASSADCO (Propagation analysis of STAFF-SA data with coherency tests) and was originally developed for the Cluster mission. It was also briefly introduced in Appendix of Santolík et al. (2002) and by Santolík and Gurnett (2002). PRASSADCO has been applied not only to Cluster data but also to multicomponent measurements from various other spacecraft missions, like Cassini (Píša et al. 2018), THEMIS (Demekhov et al. 2017), DEMETER (Hanzelka et al. 2017; Chen et al. 2017) and the Van Allen probes (Ripoll et al. 2017; Hartley et al. 2017). This technique is based on the condition of perpendicularity of the wave vector to the polarization plane of the wave's magnetic field. This leads to an overdetermined system of 6 equations for 2 unknowns. It can be solved in the least squares sense by the singular value decomposition (SVD) of a real $6 \times 3$ spectral matrix which is constructed from the measured complex $3 \times 3$ spectral matrix $\mathbf{J}$ by placing the real part of $\mathbf{J}$ above its imaginary part. SVD then yields three real singular values $\left(\lambda_{s 2} \geq \lambda_{s 1} \geq \lambda_{s 0} \geq 0\right)$, and the singular vector belonging to the smallest singular value $\left(\lambda_{s 0}\right)$ is a real unit vector pointing into the direction of the wave vector $\mathbf{k}$. This method incorporates the full spectral matrix, i.e., real and imaginary parts, for a determination of the k-direction. Thus, it uses all the available information unlike an earlier method from McPherron et al. (1972) who used only the real part, and another method proposed by Means (1972) who used only the imaginary part.

Additionally, Santolík et al. (2003) also define an ellipticity $\left(\varepsilon_{s}\right)$ and a planarity parameter $\left(F_{s}\right)$ from the singular values. They are given as

$$
\begin{gathered}
\varepsilon_{s}=\lambda_{s 1} / \lambda_{s 2}, \\
F_{s}=1-\sqrt{\lambda_{s 0} / \lambda_{s 2}} .
\end{gathered}
$$

These quantities shall assist in evaluating the results for $\mathbf{k}$. Results are considered to be unreliable if $\left|\varepsilon_{s}\right| \sim 0$, i.e., for $\sim$ linear polarization, and for small $F_{s}<1$.

Further details about ellipticity and planarity are discussed in Sect. 4. The meaning of the singular values from Santolík et al. (2003) will become clearer after taking a closer look at the SVD of the $3 \times 3$ complex spectral matrix (in Sect. 2) and its real part (in Sect. 3). In Sects. 5 and 6, results will be verified from a simulation study and observational data recorded by the THEMIS spacecraft, respectively. Section 7 finishes with a brief summary and conclusions.

\section{Singular Value Decomposition of the Complex Spectral Matrix}

SVD represents a fast numerical method for expanding a spectral matrix on an orthonormal basis of singular vectors. This iterative numerical approach (Press et al. 1992) decomposes $\mathbf{J}$ according to

$$
\mathbf{J}=\mathbf{U} \cdot \boldsymbol{\Lambda} \cdot \mathbf{V}^{H}
$$

The real matrix $\Lambda$ is a diagonal matrix with the singular values $\lambda_{i}$ along the main diagonal. All off-diagonal elements in $\boldsymbol{\Lambda}$ are zero. Thus, it is said that $\mathbf{J}$ becomes diagonalized. The complex matrices $\mathbf{U}$ and $\mathbf{V}$ are unitary, i.e., $\mathbf{U} \cdot \mathbf{U}^{H}=\mathbf{V} \cdot \mathbf{V}^{H}=\mathbf{I}$, with $\mathbf{I}$ as the identity 
matrix. Superscript $H$ indicates the complex conjugate and transpose of a matrix. The columns of $\mathbf{U}$ store the left singular vectors $\mathbf{u}_{i}$. The columns of $\mathbf{V}$ store the right singular vectors $\mathbf{v}_{i}$. They fulfill the following set of singular value problems $(i=0,1,2$ in $3 \mathrm{D})$ :

$$
\begin{gathered}
\mathbf{J} \cdot \mathbf{v}_{i}=\lambda_{i} \mathbf{u}_{i}, \\
\mathbf{J}^{H} \cdot \mathbf{u}_{i}=\lambda_{i} \mathbf{v}_{i} .
\end{gathered}
$$

Furthermore, $\mathbf{u}_{i}$ and $\mathbf{v}_{i}$ are normalized in the Euclidean norm, i.e.,

$$
\left\|\mathbf{u}_{i}\right\|=\sqrt{\mathbf{u}_{i} \cdot \mathbf{u}_{i}^{*}}=1 \quad\left(\text { same for } \mathbf{v}_{i}\right)
$$

Thus, the singular vectors constitute an orthonormal basis in the complex space.

The left and right singular vectors are at the same time the left and right eigenvectors of the matrices $\left(\mathbf{J} \cdot \mathbf{J}^{H}\right)$ and $\left(\mathbf{J}^{H} \cdot \mathbf{J}\right)$, respectively. So, they also fulfill the set of $i$ eigenvalue problems

$$
\begin{aligned}
& \left(\mathbf{J} \cdot \mathbf{J}^{H}\right) \cdot \mathbf{u}_{i}=\lambda_{i}^{2} \mathbf{u}_{i}, \\
& \left(\mathbf{J}^{H} \cdot \mathbf{J}\right) \cdot \mathbf{v}_{i}=\lambda_{i}^{2} \mathbf{v}_{i},
\end{aligned}
$$

with $\lambda_{i}^{2}$ appearing as the corresponding eigenvalues. Since $\mathbf{J}$ is a Hermitian matrix $\left(\mathbf{J}^{H}=\mathbf{J}\right)$, we get $\mathbf{J} \cdot \mathbf{J}^{H}=\mathbf{J}^{H} \cdot \mathbf{J}=\mathbf{J} \cdot \mathbf{J}$. It follows that for a Hermitian matrix, the singular value problem can be reformulated into the following set of eigenvalue problems:

$$
\begin{aligned}
& \mathbf{J} \cdot \mathbf{u}_{i}=\lambda_{i} \mathbf{u}_{i}, \\
& \mathbf{J} \cdot \mathbf{v}_{i}=\lambda_{i} \mathbf{v}_{i} .
\end{aligned}
$$

A proof from simple algebraic manipulations is provided in Appendix D. Thus, the eigenvectors of $\mathbf{J}$ are the singular vectors from SVD, and the singular values from SVD are identical to the eigenvalues of $\mathbf{J}$. For a Hermitian spectral matrix, the terms singular vectors and eigenvectors as well as singular values and eigenvalues can be used interchangeably. Furthermore, for a Hermitian $\mathbf{J}, \mathbf{u}_{i}=\mathbf{v}_{i}$ for all $\lambda_{i} \neq 0$, but in case of $\lambda_{i}=0$, Eqs. (41) and (42) are still fulfilled. Even if some $\lambda_{i}$ vanish, i.e., $\mathbf{V} \neq \mathbf{U}$, a decomposition of the Hermitian spectral matrix can be simplified to

using $\mathbf{U}$ only (see also Eq. (30)).

$$
\mathbf{J}=\mathbf{U} \cdot \boldsymbol{\Lambda} \cdot \mathbf{U}^{H}
$$

The following useful relations enable a conversion between $\mathbf{J}$ and its eigenvalues $\lambda_{i}$ :

$$
\begin{aligned}
\operatorname{tr}(\mathbf{J}) & =\sum_{i} \lambda_{i}, \\
\operatorname{tr}\left(\mathbf{J}^{k}\right) & =\sum_{i} \lambda_{i}^{k}, \\
\operatorname{det}(\mathbf{J}) & =\prod_{i} \lambda_{i} .
\end{aligned}
$$

The first relation indicates that, since all elements along the main diagonal of a spectral matrix are positive and real, also its eigenvalues are positive and real. The third relation 
confirms that, since all eigenvalues are positive and real, the determinant of the spectral matrix must also be positive and real.

\subsection{Application of the Complex SVD}

We have shown that the singular value problem that is solved by the numerical SVD algorithm can be translated into an analytical eigenvalue problem. Instead of presenting here details of the numerical SVD algorithm we demonstrate how the representative eigenvalue problem is solved analytically in order to get a better understanding of the operating principles of the complex SVD. Therefore, the spectral matrix must already be expressed in the wave-frame.

Let us consider the given situation of a partially polarized signal plus unpolarized noise. The signal shall be confined to a plane (plane wave) so that a wave-frame can be found in which all components including the $z$-coordinate are vanishing. Together with the spectral matrix for noise, the complete spectral matrix can be modeled as

$$
\begin{gathered}
\mathbf{J}=\left(\begin{array}{ccc}
J_{\mathrm{p}, x x} & J_{\mathrm{p}, x y} & 0 \\
J_{\mathrm{p}, x y}^{*} & J_{\mathrm{p}, y y} & 0 \\
0 & 0 & 0
\end{array}\right)+\left(\begin{array}{ccc}
\tilde{a} & 0 & 0 \\
0 & \tilde{a} & 0 \\
0 & 0 & 0
\end{array}\right)+\left(\begin{array}{ccc}
c & 0 & 0 \\
0 & c & 0 \\
0 & 0 & c
\end{array}\right), \\
\mathbf{J}=\left(\begin{array}{ccc}
J_{\mathrm{p}, x x} & J_{\mathrm{p}, x y} & 0 \\
J_{\mathrm{p}, x y}^{*} & J_{\mathrm{p}, y y} & 0 \\
0 & 0 & 0
\end{array}\right)+\left(\begin{array}{ccc}
a & 0 & 0 \\
0 & a & 0 \\
0 & 0 & c
\end{array}\right), \\
\mathbf{J}=\mathbf{J}_{\mathrm{p}}+\mathbf{J}_{\mathrm{n}} .
\end{gathered}
$$

The spectral matrix $\mathbf{J}$ can be depicted as a superposition of a fully polarized part $\mathbf{J}_{\mathrm{p}}$ and a noise part $\mathbf{J}_{\mathrm{n}}$, which itself is composed of the unpolarized component of the plane wave in its wavefront ( $\tilde{a})$ and general 3D isotropic noise (c), with $a=\tilde{a}+c$. The peculiarities of such a $\mathbf{J}_{\mathrm{n}}$ have already been discussed in Sect. 1, following Eq. (3). A more general overview regarding decompositions of the spectral matrix into characteristic components depending on the rank of the matrix can be found in Gil (2014).

In the wave-frame, the noise power $c$ along $z$ is directly provided as the measured quantity $J_{z z}$. In fact, if $\mathbf{J}$ is expressed in the wave-frame, the eigenvalue problem reduces from three to two dimensions. A decomposition is only required for the $2 \times 2$ sub-matrix

$$
\begin{gathered}
\mathbf{J}_{2}=\mathbf{J}_{\mathrm{p} 2}+\mathbf{J}_{n 2}, \\
\mathbf{J}_{2}=\left(\begin{array}{cc}
J_{\mathrm{p}, x x} & J_{\mathrm{p}, x y} \\
J_{\mathrm{p}, x y}^{*} & J_{\mathrm{p}, y y}
\end{array}\right)+\left(\begin{array}{ll}
a & 0 \\
0 & a
\end{array}\right) .
\end{gathered}
$$

The characteristic equation of the eigenvalue problem at hand is

$$
\begin{gathered}
\operatorname{det}\left[\mathbf{J}_{2}-\lambda \mathbf{I}\right]=0, \\
\operatorname{det}\left[\left(\begin{array}{cc}
J_{\mathrm{p}, x x}+a & J_{\mathrm{p}, x y} \\
J_{\mathrm{p}, x y}^{*} & J_{\mathrm{p}, y y}+a
\end{array}\right)-\lambda \mathbf{I}\right]=0 .
\end{gathered}
$$

This leads to the characteristic polynomial 


$$
\delta_{2} \lambda^{2}+\delta_{1} \lambda+\delta_{0}=0
$$

with the coefficients

$$
\begin{gathered}
\delta_{2}=1, \\
\delta_{1}=-\operatorname{tr}\left(\mathbf{J}_{2}\right), \\
\delta_{0}=\operatorname{det}\left(\mathbf{J}_{2}\right) .
\end{gathered}
$$

The two solutions are

$$
\lambda_{2,1}=\frac{1}{2} \operatorname{tr}\left(\mathbf{J}_{2}\right) \pm \frac{1}{2}\left[\operatorname{tr}^{2}\left(\mathbf{J}_{2}\right)-4 \operatorname{det}\left(\mathbf{J}_{2}\right)\right]^{\frac{1}{2}}
$$

If we insert

$$
\begin{gathered}
\operatorname{tr}\left(\mathbf{J}_{2}\right)=J_{\mathrm{p}, x x}+J_{\mathrm{p}, y y}+2 a, \\
\operatorname{tr}^{2}\left(\mathbf{J}_{2}\right)=4\left(J_{\mathrm{p}, x x}+a\right)\left(J_{\mathrm{p}, y y}+a\right)+\left(J_{\mathrm{p}, x x}-J_{\mathrm{p}, y y}\right)^{2}, \\
\operatorname{det}\left(\mathbf{J}_{2}\right)=\left(J_{\mathrm{p}, x x}+a\right)\left(J_{\mathrm{p}, y y}+a\right)-J_{\mathrm{p}, x y} J_{\mathrm{p}, x y}^{*},
\end{gathered}
$$

and keep in mind that $\operatorname{det}\left(\mathbf{J}_{\mathrm{p} 2}\right)=J_{\mathrm{p}, x x} J_{\mathrm{p}, y y}-J_{\mathrm{p}, x y} J_{\mathrm{p}, x y}^{*}=0$, i.e., $J_{\mathrm{p}, x y} J_{\mathrm{p}, x y}^{*}=J_{\mathrm{p}, x x} J_{\mathrm{p}, y y}$ (Fowler et al. (1967); see Appendix E), then the two possible eigenvalues are

$$
\begin{gathered}
\lambda_{2}=a+J_{\mathrm{p}, x x}+J_{\mathrm{p}, y y}, \\
\lambda_{1}=a .
\end{gathered}
$$

As can be seen, eigenvalue $\lambda_{1}$ equals the noise power $a$ inside the $[x, y]$-plane of the waveframe. The second and larger solution, $\lambda_{2}$, is a sum of $a$ and the polarized powers $J_{\mathrm{p}, x x}$ and $J_{\mathrm{p}, y y}$ along the $x$ - and $y$-directions of the wave-frame, respectively. The only purpose of $\lambda_{2}$ is to ensure that the sum of all three eigenvalues results in the correct total power, which equals $J_{\mathrm{p}, x x}+J_{\mathrm{p}, y y}+2 a+c$. Here we also see the meaning of the noise power $c$, which corresponds to the third eigenvalue. In summary, the three eigenvalues, which equal the three singular values computed from the complex SVD, are:

$$
\begin{aligned}
& \lambda_{0}=c \ldots \text { noise power along } z \\
& \lambda_{1}=a \ldots 2 \text { D- isotropic noise power inside }[x, y] \text {-plane } \\
& \lambda_{2}=a+J_{\mathrm{p}, x x}+J_{\mathrm{p}, y y} \ldots \text { ensures that } \Sigma \lambda_{i} \text { yields correct total power }
\end{aligned}
$$

The analogy between singular value problem and eigenvalue problem for a Hermitian spectral matrix enables one to get a rough idea of what the numerical SVD algorithm is actually doing. First, it tries to find a coordinate system in complex space in which $\mathbf{J}$ depicts Eq. (48) as accurately as possible. This special coordinate system is one which is able to confine the polarized part of $\mathbf{J}$ to a complex plane as efficiently as possible. The two axes inside this plane, $x$ and $y$, form two complex singular vectors. The third singular vector corresponds to the $z$-axis which is perpendicular to the plane of polarization. If a coordinate system is found which is able to entirely separate the polarized part from the noise, then the third singular vector will be entirely real. If not, i.e., if there is a small polarized component left along $z$, then the corresponding singular vector is complex as well. This can happen due to experimental inaccuracies of the spectral analysis or simply due to a superposition of many plane waves at the antenna system with slightly different directions 
of incidence (k-directions) and slightly different frequencies, i.e., in the presence of nonmonochromatic wave packets. This leads to nonzero matrix elements in the third row and third column of $\mathbf{J}$. The singular value $\lambda_{0}$ will then contain not only noise but also the power of the residual polarized component which is left along the $z$-direction, i.e.,

$$
\lambda_{0}=c+J_{\mathrm{p}, z z} \ldots \text { total power along } z
$$

After establishing the optimum wave-frame in complex space and expressing $\mathbf{J}$ in it, the power along the $z$-axis is identified as the first singular value. Then, the $2 \mathrm{D}$ sub-matrix $\mathbf{J}_{2}$ is solved for the other two singular values inside the $[x, y]$-plane of the wave-frame.

An issue which has to be handled carefully is the assignment of singular values to the three axes. If one assumes the presence of 3D isotropic noise (ambient and/or instrumental) plus a partially polarized plane wave, as depicted in Eq. (47), then the noise power $a$ will contain a sum of the isotropic noise component $(=c)$ and the unpolarized power from the partially polarized signal ( $\tilde{a})$. Thus, $a=\tilde{a}+c$ will exceed $c$, and it seems to be safe to assign the smallest singular value directly to $c$, i.e., $\lambda_{0}=c$, and the medium singular value to $a$, i.e., $\lambda_{1}=a$, yielding $\lambda_{0} \leq \lambda_{1} \leq \lambda_{2}$. However, if there is a strong residual polarized component left along the $z$-direction, as discussed above, then it is possible that $\lambda_{0}=c+J_{\mathrm{p}, z z}$ exceeds $\lambda_{1}=a$, yielding $\lambda_{1} \leq \lambda_{0} \leq \lambda_{2}$. Thus, an assignment of the two smallest singular values to axes directions cannot be done according to their absolute values alone. This problem is discussed further in Sect. 3, after examining the SVD of the real part of the spectral matrix.

\section{Singular Value Decomposition (SVD) of the Real Part of J}

The eigenvalue problem shall now be formulated for the real part of the complex spectral matrix, i.e.,

$$
\operatorname{det}[\Re\{\mathbf{J}\}-\lambda \mathbf{I}]=0 .
$$

This approach dates back to McPherron et al. (1972). As for the complex case, the first eigenvalue can be directly identified with the noise power along the $z$-direction, i.e., $\lambda_{r 0}=c$. Under more realistic conditions with a polarized component left along $z, \lambda_{r 0}$ becomes the total power along $z$. Subscript $r$ shall indicate that $\lambda$ belongs to $\mathfrak{R}\{\mathbf{J}\}$. The other two eigenvalues are solutions for the $2 \times 2$ sub-matrix $\mathfrak{R}\left\{\mathbf{J}_{2}\right\}$ in the $[x, y]$-plane of the wave-frame, leading to the characteristic equation

$$
\operatorname{det}\left[\left(\begin{array}{cc}
J_{\mathrm{p}, x x}+a & \mathfrak{R}\left\{J_{x y}\right\} \\
\mathfrak{R}\left\{J_{x y}\right\} & J_{\mathrm{p}, y y}+a
\end{array}\right)-\lambda_{r} \mathbf{I}\right]=0 .
$$

Now, $\mathbf{J}_{2}$ shall be expressed in a coordinate system which is rotated by the angle $\tau$ (see Eq. (11)) around the $z$-axis so that the new $x$-axis coincides with the semi-major axis of the polarization ellipse and the new $y$-axis coincides with the semi-minor axis of the polarization ellipse. In this main-axis frame, $\mathfrak{R}\left\{J_{x y}\right\}=0$. Since the noise power $a$ is isotropic in the $[x, y]$-plane, it is unaffected by a rotation around the $z$-axis, i.e., $a$ is also valid along the main axes of the polarization ellipse. In the main-axis frame, Eq. (65) becomes

$$
\operatorname{det}\left[\left(\begin{array}{cc}
J_{\mathrm{p}, x x}^{\prime}+a & 0 \\
0 & J_{\mathrm{p}, y y}^{\prime}+a
\end{array}\right)-\lambda_{r} \mathbf{I}\right]=0 .
$$

$\mathrm{A}^{\prime}$-symbol shall indicate that components are given in the main-axis frame now. The characteristic polynomial is 


$$
\lambda_{r}^{2}-\lambda_{r}\left(J_{\mathrm{p}, x x}^{\prime}+J_{\mathrm{p}, y y}^{\prime}+2 a\right)+\left(J_{\mathrm{p}, x x}^{\prime}+a\right)\left(J_{\mathrm{p}, y y}^{\prime}+a\right)=0,
$$

with the two solutions

$$
\begin{aligned}
& \lambda_{r 2}=a+J_{\mathrm{p}, x x}^{\prime}, \\
& \lambda_{r 1}=a+J_{\mathrm{p}, y y}^{\prime} .
\end{aligned}
$$

So, applying the SVD to $\mathfrak{R}\{\mathbf{J}\}$ does the following: it establishes a wave-frame in which the singular vectors $\mathbf{u}_{r 2}$ (for $\lambda_{r 2}$ ) and $\mathbf{u}_{r 1}$ (for $\lambda_{r 1}$ ) point along the semi-major and semi-minor axis of the polarization ellipse, respectively. The third singular vector (for $\lambda_{r 0}$ ) corresponds to the $z$-axis, which is perpendicular to the plane of the wavefront. In contrast to the complex SVD, the three singular vectors are entirely real. Thus, if $\mathbf{J}$ is established with the wave's magnetic field, the direction of $\mathbf{u}_{r 0}$ provides an estimate for the direction of the wave vector $\mathbf{k}$.

In summary, SVD of $\mathfrak{R}\{\mathbf{J}\}$ yields the following products:

$$
\begin{aligned}
\lambda_{r 0}= & c+J_{\mathrm{p}, z z} \ldots \text { total power along } z \\
\lambda_{r 1}= & a+J_{\mathrm{p}, y y}^{\prime} \ldots \text { total power along semi- minor axis of pol. ellipse } \\
\lambda_{r 2}= & a+J_{\mathrm{p}, x x}^{\prime} \ldots \text { total power along semi- major axis of pol. ellipse } \\
& \left.\mathbf{u}_{r 0} \ldots \text { wave vector direction (in case } \mathbf{J}=\mathbf{J}(\mathbf{B})\right)
\end{aligned}
$$

The three singular values can be visualized as spanning an ellipsoid similar to the inertia ellipsoid defined by Dennis (2004) from $\mathfrak{R}\{\mathbf{J}\}$. The coordinate system is always established such that $\lambda_{r 0}$ is the smallest singular value, i.e., $0 \leq \lambda_{r 0} \leq \lambda_{r 1} \leq \lambda_{r 2}$. In comparison with the complex SVD from Sect. 2.1, we also see that $\lambda_{r 0}=\lambda_{0}$. Since $\lambda_{0}$ can be less than $\lambda_{1}$ or greater than $\lambda_{1}$, a comparison to $\lambda_{r 0}$ from real SVD can help to resolve this problem. If $\lambda_{r 0}<\lambda_{1}$, then $\lambda_{0}$ is indeed the smallest singular value from complex SVD. If $\lambda_{r 0}>\lambda_{1}$, then $\lambda_{0}$ corresponds to the medium singular value from complex SVD.

The singular values of $\mathfrak{R}\{\mathbf{J}\}$ do differ not only from those of the complex $\mathbf{J}$ (except $\lambda_{r 0}$ ), but also from those of the real $6 \times 3$ spectral matrix from Santolík et al. (2003), which are labeled $\lambda_{s 0}, \lambda_{s 1}$ and $\lambda_{s 2}$ in Sect. 1.2. The latter represent power along each axis of the polarization ellipsoid only in the case of noise, but for a purely polarized signal $\lambda_{s 1}$ and $\lambda_{s 2}$ are proportional to the amplitude along the semi-minor and semi-major axis of the polarization ellipse, respectively, implying expression (33) for the ellipticity (see Appendix in Santolík et al. 2003). Therefore, the total power is not always given by $\sum_{i} \lambda_{s i}$, and $\lambda_{s i}$ cannot be used to compute a degree of polarization. However, the directions of singular vectors from Santolík et al. (2003) do point along $\mathbf{k}$ and along the main axes of the polarization ellipse as well. Furthermore, those directions are more accurate estimates than directions computed from $\mathfrak{R}\{\mathbf{J}\}$ because information contained in the imaginary part is not neglected.

\section{Revisiting Ellipticity, Planarity and Degree of Polarization}

A combination of singular values from SVD of the complex spectral matrix $\mathbf{J}$ and of $\Re\{\mathbf{J}\}$ enables a separation of polarized signal and noise inside the $[x, y]$-plane of the wave-frame. The power of the polarized components along the main axes of the polarization ellipse can be estimated as

$$
J_{\mathrm{p}, x x}^{\prime}=\lambda_{r 2}-\lambda_{1}
$$




$$
J_{\mathrm{p}, y y}^{\prime}=\lambda_{r 1}-\lambda_{1},
$$

with $\lambda_{1}$ as the $2 \mathrm{D}$-isotropic noise power inside the plane of the wavefront. $J_{\mathrm{p}, x x}^{\prime}$ and $J_{\mathrm{p}, y y}^{\prime}$ can be used to compute the ellipticity of the polarization ellipse according to

$$
\varepsilon_{\mathrm{p}}=\frac{\sqrt{J_{\mathrm{p}, y y}^{\prime}}}{\sqrt{J_{\mathrm{p}, x x}^{\prime}}}=\sqrt{\frac{\lambda_{r 1}-\lambda_{1}}{\lambda_{r 2}-\lambda_{1}}} .
$$

Note that amplitudes, i.e., square-roots of power, are required here. Subscript $p$ shall indicate that only the polarized components are utilized. This definition of ellipticity differs from Eq. (33) insofar that it is noise-free. A signed ellipticity (negative for LH, positive for RH) is obtained by adopting the sign of the cross-spectral component $\mathfrak{\Im}\left\{J_{x y}\right\}$, preferably after rotating $\mathbf{J}$ into a coordinate system with a $z$-axis aligned with the ambient magnetic field.

The planarity parameter $F_{s}$ from Eq. (34) as defined by Santolík et al. (2003) is a comparison between the maximum amplitude inside the wavefront and the amplitude along the minimum variance direction (k-direction). It can as well be calculated with the singular values of $\mathfrak{R}\{\mathbf{J}\}$, i.e., $F=1-\sqrt{\lambda_{r 0} / \lambda_{r 2}} . F \approx 1$ characterizes the presence of a single plane wave, and $F<1$ indicates a violation of this assumption. Note that $F=1$ is hardly fulfilled unless noise and polarized power along $z$ vanish completely.

In case there is noise and a polarized component left along the $z$-direction, both real and complex SVD do not provide any indication on how the polarized component $J_{\mathrm{p}, z z}$ can be separated from the noise $c . J_{\mathrm{p}, z z}$ and $c$ both contribute to $\lambda_{r 0}$ (or $\lambda_{0}$, or $\lambda_{s 0}$ ), but their shares remain unknown. Noise and polarized signal can only be separated inside the $[x, y]$-plane of the wave-frame, but not along the $z$-direction. If a noise-biased $\lambda_{r 0}\left(\right.$ or $\left.\lambda_{s 0}\right)$ has to be used for defining a planarity, then the quantity representing the maximum amplitude inside the wavefront $\left(\lambda_{r 2}\right.$, or $\left.\lambda_{s 2}\right)$ should contain noise as well in order to yield a more constructive comparison of amplitudes. Thus, definition (34) from Santolík et al. (2003) is a reasonable estimate for the wave's planarity, even if it is not noise-free.

The degree of polarization, which specifies the ratio of polarized power to total power, can be found with the singular values from complex SVD as follows:

$$
\begin{aligned}
& D_{\mathrm{p}}=\frac{\operatorname{tr}\left(\mathbf{J}_{\mathrm{p}}\right)}{\operatorname{tr}(\mathbf{J})}=\frac{\operatorname{tr}(\mathbf{J})-\operatorname{tr}\left(\mathbf{J}_{\mathrm{n}}\right)}{\operatorname{tr}(\mathbf{J})} \\
= & \frac{\left(\lambda_{0}+\lambda_{1}+\lambda_{2}\right)-\left(\lambda_{0}+2 \lambda_{1}\right)}{\lambda_{0}+\lambda_{1}+\lambda_{2}} \\
= & \frac{\lambda_{2}-\lambda_{1}}{\lambda_{0}+\lambda_{1}+\lambda_{2}} \equiv D_{\mathrm{p} 3 e} .
\end{aligned}
$$

It turns out that incorporating all available information obtained from SVD of the complex spectral matrix yields a formulation for the degree of polarization which is identical to the one derived by Ellis et al. (2005) (see Eq. (32) for comparison). This formulation takes into account that noise might not be strictly isotropic but that an additional noise component might be added from a partially polarized plane wave. 
For strictly isotropic noise, $a=c$, and $\lambda_{0}=\lambda_{1}$ in the presence of a single plane wave. Then, inserting $\operatorname{tr}\left(\mathbf{J}_{\mathrm{n}}\right)=3 \lambda_{0}$ into Eq. (73) yields

$$
D_{\mathrm{p} 3 i}=1-\frac{3 \lambda_{0}}{\lambda_{0}+\lambda_{1}+\lambda_{2}} .
$$

$D_{\mathrm{p} 3 i}$ (subscript $i$ stands for isotropic) is also called degree of directionality (Gil 2007) because it neglects the noisy component from a partially polarized plane wave.

\section{Simulation of Polarization Parameters}

In this section, we are going to model spectral matrices for noise and polarized signals. Subsequently, singular values from real and complex SVD will be combined to several polarization parameters which have already been discussed in the previous sections. These parameters can be compared to their preset values from the model in order to verify their accuracy. Furthermore, the performance of polarization parameters as a function of signalto-noise ratio (SNR) will be analyzed. The SNR is simply defined as

$$
\mathrm{SNR}=\frac{\operatorname{tr}\left(\mathbf{J}_{s}\right)}{\operatorname{tr}\left(\mathbf{J}_{\mathrm{n}}\right)}
$$

with $\mathbf{J}_{\mathrm{n}}$ as the noise spectral matrix and $\mathbf{J}_{s}$ as the spectral matrix for the (partially) polarized signal.

\subsection{Simulations for Singular Values, Degree of Polarization and Ellipticity}

Two spectral matrices for two different scenarios are established here: (a) a fully polarized plane wave plus noise and (b) a partially polarized plane wave plus noise. The first scenario has the following spectral matrix in the wave-frame:

$$
\mathbf{J}=\left(\begin{array}{ccc}
J_{\mathrm{p}, x x} & J_{\mathrm{p}, x y} & 0 \\
J_{\mathrm{p}, x y}^{*} & J_{\mathrm{p}, y y} & 0 \\
0 & 0 & 0
\end{array}\right)+\left(\begin{array}{ccc}
c & 0 & 0 \\
0 & c & 0 \\
0 & 0 & c
\end{array}\right) .
$$

The second scenario is the one already depicted in Eq. (47), i.e., a partially polarized plane wave plus isotropic noise. This is modeled in the wave-frame as

$$
\mathbf{J}=\left(\begin{array}{ccc}
J_{\mathrm{p}, x x}+\tilde{a} & J_{\mathrm{p}, x y} & 0 \\
J_{\mathrm{p}, x y}^{*} & J_{\mathrm{p}, y y}+\tilde{a} & 0 \\
0 & 0 & 0
\end{array}\right)+\left(\begin{array}{ccc}
c & 0 & 0 \\
0 & c & 0 \\
0 & 0 & c
\end{array}\right),
$$

with $\tilde{a}$ as the unpolarized power in the partially polarized signal. For the computation of the signal-to-noise ratio from Eq. (76), $\mathbf{J}_{\mathrm{n}}$ equals the second matrix on the right-hand sides of Eqs. (77) and (78). $\mathbf{J}_{s}$ of the signal is modeled by the first matrix on the right-hand sides of Eqs. (77) and (78).

The components of $\mathbf{J}_{\mathrm{p}}$, i.e., of the matrix for the fully polarized part, are computed in the wave-frame for fixed values of $\tau\left(=30^{\circ}\right)$ and $\varepsilon(=0.30)$, which specify tilt and shape of the polarization ellipse. The Stokes parameter $S$ of the signal $\left(=\operatorname{tr}\left(\mathbf{J}_{s}\right)\right)$ is set to be logarithmically increasing from $10^{-3}$ to $10^{3}$, while the total noise power is kept at a constant value of 
$1\left(\operatorname{tr}\left(\mathbf{J}_{\mathrm{n}}\right)=1, c=1 / 3\right)$. This shall mimic a broad range of signal-to-noise ratios. The noise power $\tilde{a}$ of the partially polarized signal is adjusted such that $D_{\mathrm{p}}=0.6(60 \%$ polarized $)$. From $S=\operatorname{tr}\left(\mathbf{J}_{S}\right)=\left(J_{\mathrm{p}, x x}+J_{\mathrm{p}, y y}\right)+2 \tilde{a}=0.6 S+(1-0.6) S$, one gets $\tilde{a}=(1-0.6) S / 2$.

Figure 1 summarizes our results for the two simulated scenarios: for a $100 \%$ polarized signal on the left-hand side (a-c) and for a $60 \%$ polarized signal on the right-hand side (d-f). Figure 1a, d shows the singular values from complex SVD as a function of SNR. As can be seen, the smallest singular value $\lambda_{0}$ (blue line) always matches the preset noise power $c=1 / 3$. For the $100 \%$ polarized signal, the green dashed line for $\lambda_{1}$ coincides with $\lambda_{0}$, but for the partially polarized signal $\lambda_{1}=\tilde{a}+c$. As the signal power $S$ is increasing from left to right, so do $\tilde{a}$ and $\lambda_{1}$. $\lambda_{2}$ (red line), the largest singular value, contains all the remaining power which is needed to end up at the correct total power of $S+1$.

Figure $1 \mathrm{~b}$, e shows the behavior of various definitions of the degree of polarization as a function of SNR. Results are compared to the true degree of polarization $\left(D_{\mathrm{p}, \text { true }}\right)$ which is marked by a black dashed-dotted line. $D_{\mathrm{p}, \text { true }}$ is known from the initial setup of the model. $D_{\mathrm{p} 2}$ (blue), $D_{\mathrm{p} 3}$ (green) (Samson and Olson 1980), $D_{\mathrm{p} 3 e}$ (red) (Ellis et al. 2005) and $D_{\mathrm{p} 3 i}$ (orange) (Gil 2007) are computed from Eqs. (20), (29), (74) and (75), respectively. For a $100 \%$ polarized signal, almost all definitions of the degree of polarization match the true value, except $D_{\mathrm{p} 2}$, which is ignoring $c$ along the $z$-direction and is thus underestimating the noise. The colored lines for $D_{\mathrm{p} 3}$ and $D_{\mathrm{p} 3 i}$ are hidden underneath the red line for $D_{\mathrm{p} 3 e}$. It is also obvious that due to the depolarizing influence of the noise, the preset value for $D_{\mathrm{p}}$ is only reached for a sufficiently strong signal with a SNR $>10$.
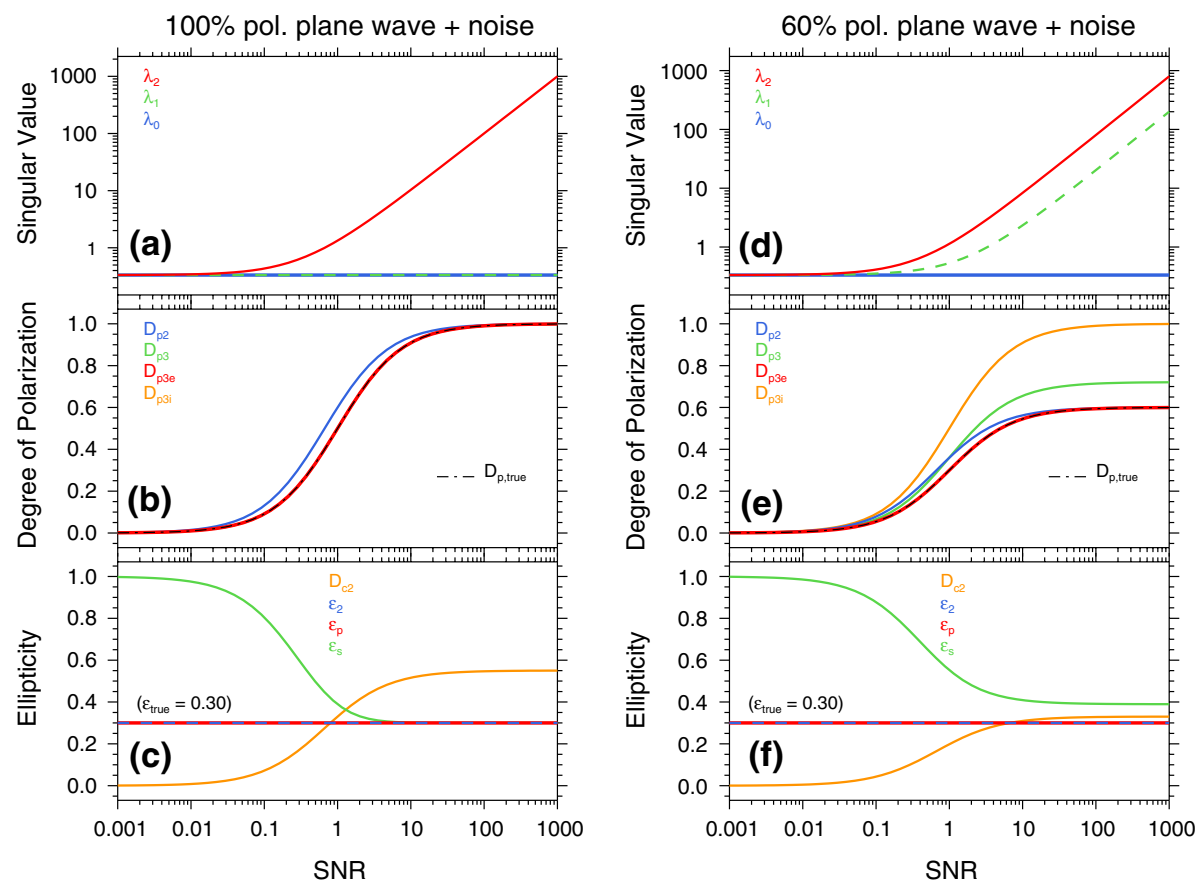

Fig. 1 a, $\mathbf{d}$ Singular values from complex SVD, $\mathbf{b}, \mathbf{e}$ four definitions of the degree of polarization, and $\mathbf{c}, \mathbf{f}$ three definitions for ellipticity and the degree of circular polarization as a function of signal-to-noise ratio (SNR) 
In the case of the partially polarized signal, there are clear deviations visible with respect to $D_{\mathrm{p} \text {,true }} . D_{\mathrm{p} 3 i}$ (orange line in Fig. 1e) ignores contributions to noise from the unpolarized component of the plane wave. A very strong signal with SNR $\gg 10$ is always detected as almost $100 \%$ polarized. $D_{\mathrm{p} 3}$ (green) from Samson and Olson (1980) is overestimating $D_{\mathrm{p} \text {,true }}$ just slightly. In fact it can be shown that $D_{\mathrm{p} 3}=0.5\left(D_{\mathrm{p} 3 i}^{2}+3 D_{\mathrm{p} 3 e}^{2}\right)^{1 / 2}(\mathrm{Gil}$ and San José 2010). Only $D_{\mathrm{p} 3 e}$ from Ellis et al. (2005) (red) and $D_{\mathrm{p} 2}$ (blue) at high SNR are able to identify the unpolarized component of the partially polarized plane wave correctly.

Figure 1c, f displays the 2D degree of circular polarization $\left(D_{c 2}\right)$ and three definitions for the ellipticity $\left(\varepsilon_{2}, \varepsilon_{\mathrm{p}}, \varepsilon_{s}\right)$ as a function of SNR. $D_{c 2}$ (orange), $\varepsilon_{2}$ (blue), $\varepsilon_{\mathrm{p}}$ (red) and $\varepsilon_{s}$ (green) are computed from Eqs. (16), (13), (72) and (33), respectively. The preset ellipticity of 0.30 is reproduced by $\varepsilon_{2}$ and $\varepsilon_{\mathrm{p}}$. Only these two definitions manage to split the noise correctly from the polarized component of the plane wave. $\varepsilon_{s}$ is overestimating the true ellipticity, especially at low SNR, because its computation involves not only the polarized signal but also noise. $\left|\varepsilon_{s}\right|$ is always approaching 1 at low SNR for isotropic noise. It should be mentioned that our model represents an idealized case. In reality, such a clear separation into polarized part and noise is not always possible. For low SNR, $\varepsilon_{2}$ and $\varepsilon_{\mathrm{p}}$ would begin to fluctuate around the true value. We want to emphasize that all the ellipticity parameters should be interpreted with caution at $\mathrm{SNR}<10$.

\subsection{Simulations for Planarity and Wave Normal Angle}

Figure 2 presents a dependence of two particular parameters on SNR: the planarity and the wave normal angle $\theta_{k}$, i.e., the polar angle of $\mathbf{k}$ in a frame different from the wave-frame. Both parameters are computed according to the method of Santolík et al. (2003): the planarity is from Eq. (34), and $\theta_{k}$ is from the singular vector belonging to the smallest singular value $\lambda_{s 0}$.

In principle, the planarity can be simulated as is done in the previous section, i.e., by starting from a modeled spectral matrix. On the other hand, a simulation of a dependence of $\theta_{k}$ on SNR requires a different approach because changes in $\theta_{k}$ can only be introduced through an additional polarized component. These changes are supposed to be random fluctuations which originate from a $\mathbf{J}_{\mathrm{n}}$ with a weak randomly polarized component. Thus, a more realistic approach for establishing such an "imperfect" $\mathbf{J}_{n}$ is to start from modeling the waveforms in the time domain.
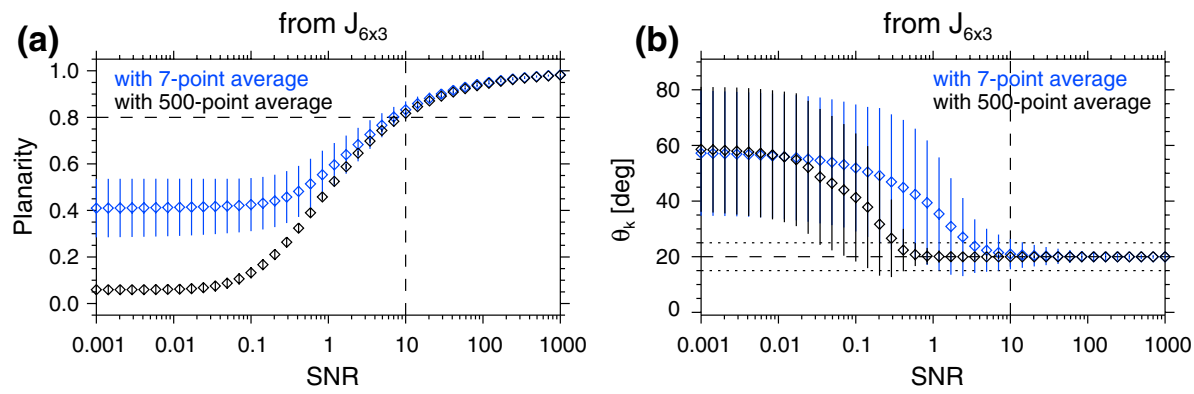

Fig. 2 a Planarity and $\mathbf{b}$ wave normal angle $\theta_{k}$ as a function of SNR. Mean values are indicated by diamond symbols and standard deviations $( \pm \sigma)$ are drawn as vertical bars 
The three waveforms for noise (in $x$-, $y$ - and $z$-directions) are modeled with amplitude values which are drawn from a random Gaussian distribution, resulting in isotropic white noise ( $\sim$ same noise power for all frequencies). The noise waveforms are superposed on that for a monochromatic harmonic wave with a fixed frequency, which we call the signal. The three signal waveforms are established such that they generate an elliptically polarized plane wave with ellipticity $=0.30, \tau=30^{\circ}, \theta_{k}=20^{\circ}$ and $\phi_{k}=0^{\circ}$.

Over successive runs, the Stokes parameter $S$ of the polarized signal is set to be increasing logarithmically, but the intensity of the noise level, i.e., the variance $\sigma^{2}$ of noise amplitudes, is kept constant. Forty runs with signal-to-noise ratios from $10^{-3}$ to $10^{3}$ are generated. For each run, the modeled waveforms of the signal and noise are superposed and Fourier-transformed (FFT). Auto-spectral powers and cross-spectral powers are computed from the complex amplitudes and averaged over frequency (rectangular weighting function). They are compiled to spectral matrices of the form presented in Eq. (1), which are finally subject to SVD analysis.

In order to achieve a more statistically significant result, the sequence of 40 runs is repeated 100 times, and each time new random series for the three noise waveforms are computed ( $\sigma^{2}$ stays constant). So, for each of the 40 SNR-bins along the $x$-axis in Fig. 2 , a set of 100 values is available for computing a mean (indicated by a diamond symbol) and a standard deviation (indicated by a vertical bar).

As can be seen in Fig. 2a, the mean planarity smoothly approaches a value of one as the SNR is increasing. As mentioned in the previous section, SNR = 10 can be considered as a threshold above which polarization parameters can be considered to be computed correctly. SNR $>10$ approximately corresponds to a planarity $>0.8$ (marked by dashed lines in Fig. 2a).

The black and blue diamond symbols in Fig. 2 belong to two different strategies of spectral averaging. The black color means that auto-spectral and cross-spectral powers are averaged (rectangular weighting) over all available discrete frequencies (500) between zero and the Nyquist frequency $\left(f_{N y}\right)$. The blue color means that spectral averaging is performed only over 7 frequency channels around the signal's frequency $\left(=0.1 f_{N y}\right)$. As can be seen, a low number of frequency channels for spectral averaging prevents the planarity from reaching zero at the smallest SNR. In fact, here we see the planarity of the randomly polarized component in our modeled noise rather than the planarity of the modeled signal.

Figure $2 \mathrm{~b}$ shows the variability of $\theta_{k}$ with SNR. The preset value of $\theta_{k}=20^{\circ}$ (marked by a horizontal dashed line) is very well reproduced at SNR $>10$, not only for the 500-channel average but also for the 7-channel average. In the limiting case of a 7-channel average, errors in $\theta_{k}$ can be expected to be less than $\sim 5^{\circ}$ at $\mathrm{SNR} \geq 10$, or at planarities $\geq 0.8$. $\mathrm{A} \pm 5^{\circ}$ error band around $20^{\circ}$ is indicated in Fig. 2 b by horizontal dotted lines. At SNR $\ll 1$, the mean $\theta_{k}$ approaches $\sim 57^{\circ}$. This corresponds to the mean value of $\sin (\theta)$ for $\theta$ between $0^{\circ}$ and $180^{\circ}$, resulting from the situation when all wave vector directions are obtained with equal probability density.

The number of frequency channels over which spectral averaging is performed influences how much a non-vanishing polarized component in $\mathbf{J}_{\mathrm{n}}$ becomes suppressed. Larger numbers result in a more accurate noise-like behavior for $\mathbf{J}_{\mathrm{n}}$, but also blur the spectrum of a signal which is short-lived and/or very narrowband in frequency. A number of 7 for averaging is definitely settled at the lower limit, mainly aiming at high spectral resolution, but it still keeps errors in polarization parameters within reasonable bounds. However, a careful selection of data with regard to SNR or planarity is inevitable if results for the polarization are to be interpreted. 


\section{Test with THEMIS Spacecraft Data}

The THEMIS mission consists of five identical satellites which are orbiting Earth on nearequatorial orbits since the beginning of 2007 (Angelopoulos 2008). Three search coil magnetometers (Le Contel et al. 2008) and three electric antennas (Bonnell et al. 2008) are able to measure fields of electromagnetic waves in a high-resolution burst-mode in which magnetic and electric waveforms are sampled at a rate of $8192 \mathrm{~Hz}$. Waveforms are windowed with a 4-term Blackman-Harris window function (75\% overlapping) in the time domain and Fourier-transformed into the frequency domain. Averaging is performed for seven neighboring frequency channels and seven successive windows in time (Welch 1967). The Hamming function provides the weight factors for averaging.

Figure 3a shows part of a dynamic spectrogram from a burst-mode snapshot recorded by THEMIS-D during November 9, 2008. Colors indicate the magnetic intensity as the sum of auto-power spectral density along all three directions, equaling the trace of the magnetic spectral matrix, i.e., $\left\langle B B^{*}\right\rangle=\left\langle B_{x} B_{x}^{*}\right\rangle+\left\langle B_{y} B_{y}^{*}\right\rangle+\left\langle B_{z} B_{z}^{*}\right\rangle$. Figure $3 \mathrm{~b}-\mathrm{d}$ presents profiles of singular values as a function of time, computed from complex $\operatorname{SVD}\left(\lambda_{0}, \lambda_{1}\right)$ and real SVD $\left(\lambda_{r 0}, \lambda_{r 1}, \lambda_{r 2}\right)$ of the magnetic spectral matrix at three selected frequencies.

Figure $3 \mathrm{~b}$ is from a cut along $2204 \mathrm{~Hz}$ including mainly background noise. All singular values are fluctuating around a noise level of approximately $10^{-9} \mathrm{nT}^{2} \mathrm{~Hz}^{-1}$ which is indicated by a horizontal dashed line. Some small differences between $\lambda_{r 0}$ (yellow), $\lambda_{r 1}$ (orange) and $\lambda_{r 2}$ (red) indicate a non-vanishing polarized component which emerges naturally from random fluctuations. The wave-frame found via SVD is based on this randomly polarized component which is finally responsible for the random fluctuations seen in the singular values.

Figure $3 \mathrm{c}$ shows profiles of singular values from a cut along $1503 \mathrm{~Hz}$ through a band of polarized emission between 1100 and $1800 \mathrm{~Hz}$. With $\lambda_{r 0}$ being about one order of magnitude smaller than $\lambda_{r 2}$, and $\lambda_{r 2} \approx \lambda_{r 1}$, this radiation possesses not only high planarity $(\sim 0.8)$ but is also strongly circularly polarized. It belongs to the so-called whistler-mode which is propagating below the local electron cyclotron frequency $\left(f_{c, e}\right)$ and the local plasma frequency. The polarization parameters will be discussed in more detail below, in reference to Fig. 4. The singular value $\lambda_{1}$ (green) contains isotropic noise $(c)$ and the noisy part of the signal $(\tilde{a})$, i.e., $\lambda_{1}=c+\tilde{a}$. It always remains close to the background noise level of $10^{-9} \mathrm{nT}^{2} \mathrm{~Hz}^{-1}$ found at $2204 \mathrm{~Hz}$, indicating that $\tilde{a} \approx 0$ and $\lambda_{1} \approx c$. The singular value $\lambda_{0}$ turns out to be larger than $\lambda_{1}$ due to an additional contribution to $\lambda_{0}$ from polarized power along $z\left(J_{\mathrm{p}, z z}\right)$.

The banded whistler-mode around $1503 \mathrm{~Hz}$ appears to be quite uniform in time and frequency, except a few more intense drifting structures, which are called chorus emissions. Chorus is showing up more prominently at frequencies below $1000 \mathrm{~Hz}$, which is below one half of the local $f_{c, e}$ (lower band). Eight distinct chorus bursts with positive sweep rates are visible. Corresponding profiles of singular values at $801 \mathrm{~Hz}$ are displayed in Fig. 3d. With $\lambda_{r 1} \approx \lambda_{r 2}$, chorus also belongs to the circularly polarized whistler-mode. Whenever a chorus signal is intersected, $\lambda_{0}$ clearly exceeds $\lambda_{1}$, meaning that a nonzero $J_{\mathrm{p}, z z}$ is contributing significantly to $\lambda_{0}$. The assumption of the presence of a plane wave is still reasonably fulfilled because $\lambda_{0}$ remains more than one order of magnitude below $\lambda_{r 2}$, especially in the center of each chorus element (planarity $\sim 0.9$; see also Fig. $4 \mathrm{a}$ ). Whereas for the banded whistler-mode in Fig. $3 c \lambda_{1}$ keeps fluctuating around the noise level $\left(\lambda_{1} \approx c\right)$, the situation seems to be different for chorus. Figure $3 \mathrm{~d}$ shows a clear increase in $\lambda_{1}$ at times when a chorus element gets intersected. Since $c$ is assumed to be unaffected by the chorus signal, an 
(a)
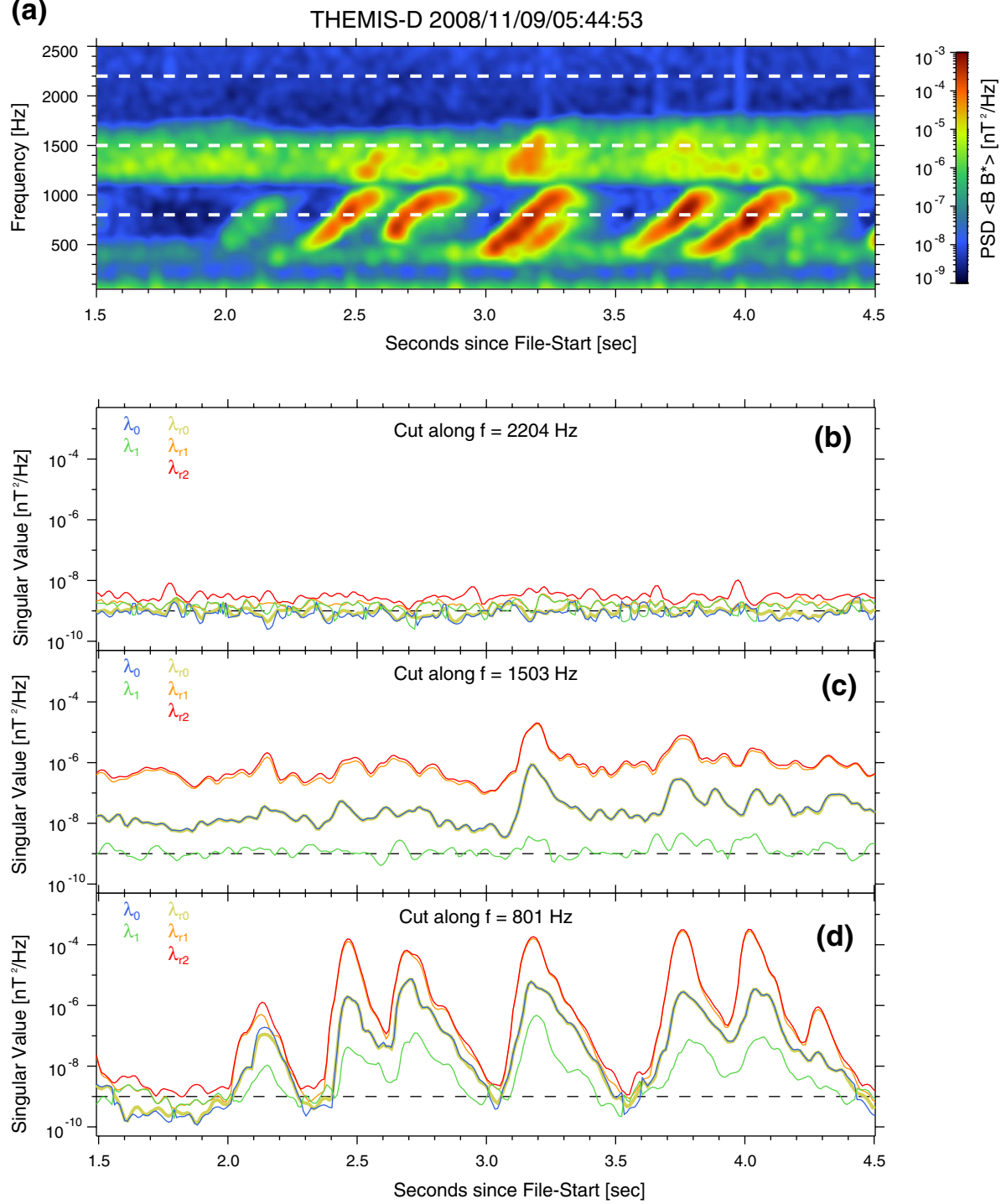

Fig. 3 THEMIS-D observations of chorus emissions in the vicinity of Earth from November 9, 2008. a Total magnetic auto-power spectral density, and singular values from complex and real SVD along spectral cuts at b $2204 \mathrm{~Hz}, \mathbf{c} 1503 \mathrm{~Hz}$ and d $801 \mathrm{~Hz}$

increase in $\lambda_{1}$ must come from $\tilde{a}$, i.e., the unpolarized part of the partially polarized chorus signal. Nevertheless, $\lambda_{1}$ remains smaller than $\lambda_{0}$.

This example of THEMIS measurements demonstrates that in the presence of a highly polarized signal, $\lambda_{r 0} \leq \lambda_{r 1} \leq \lambda_{r 2}$ is trivially fulfilled, but assuming $\lambda_{0} \leq \lambda_{1}$ is usually not justified.

The singular values from the complex and real SVD can be combined to calculate various polarization parameters: the planarity from Equation (34), several definitions of the degree of 


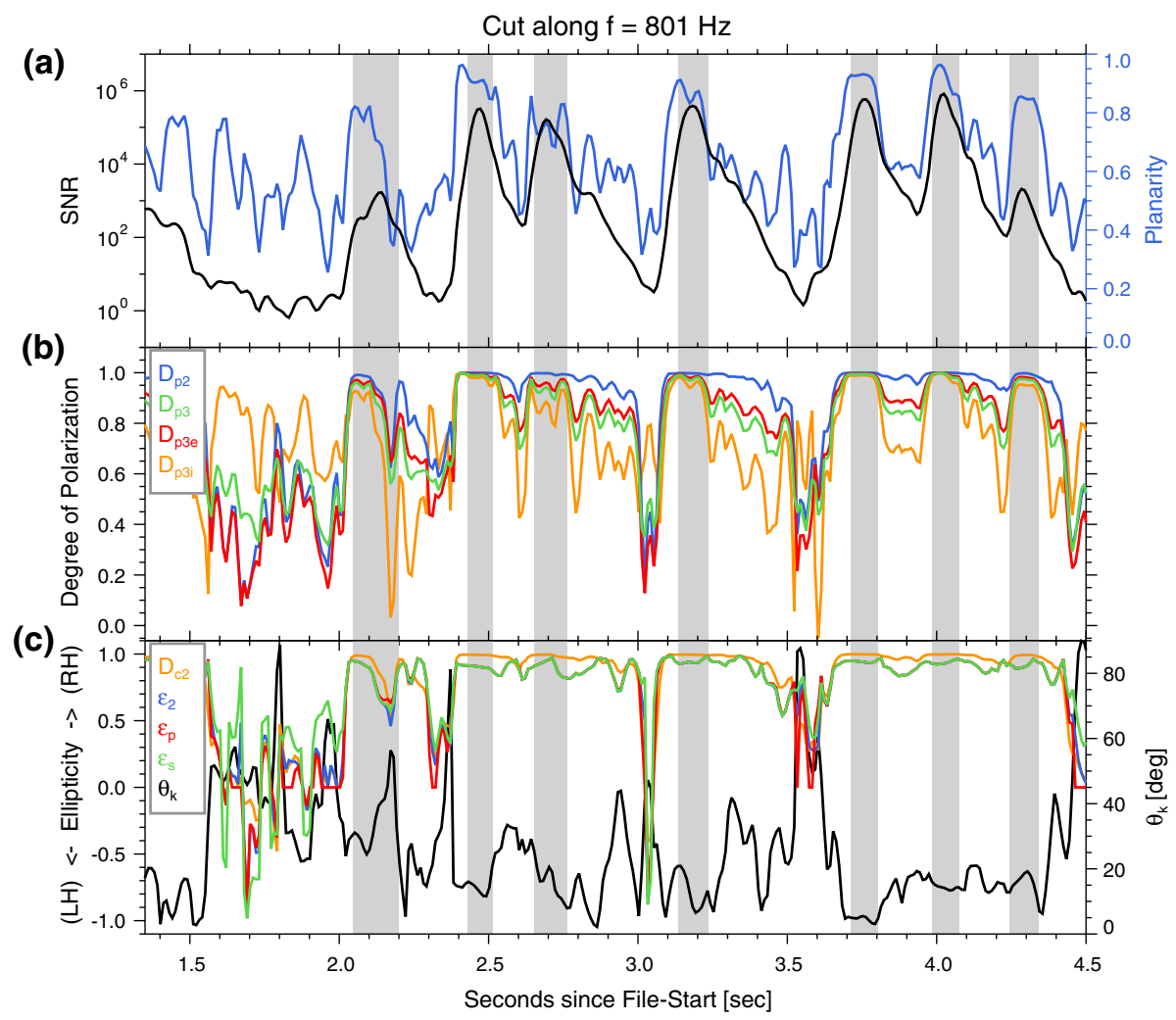

Fig. 4 Profiles for SNR and various polarization parameters from THEMIS-D measurements (see Figure $3 \mathrm{a}$ ) at a frequency of $801 \mathrm{~Hz}$. Gray vertical bars highlight time intervals when chorus elements are intersected

polarization (see Eqs. (20), (29), (74), (75)), several definitions of the ellipticity (see Eqs. (13), (72), (33)) and the degree of circular polarization (see Eq. (16)). Furthermore, we also take a brief look at measured wave normal angles. Results are summarized in Fig. 4 for a spectral cut along a single frequency of $801 \mathrm{~Hz}$, i.e., through the lower band chorus elements. Used colors for profiles and the nomenclature are identical to Fig. 1.

Gray vertical bars in Fig. 4 indicate time intervals when chorus elements are intersected. They are drawn around the peaks found in the profile of signal-to-noise ratio (SNR; black curve in Fig. 4a) using a threshold of peak level minus one order of magnitude. In contrast to the case of synthetic data, it is impossible for real measurements to determine the exact noise level from the singular values in the presence of a strong signal due to violations of the plane wave assumption, even if these violations are small. This issue was discussed in the paragraphs above. Thus, instead of computing the SNR from the singular values, as done in Sect. 5, we simply assume a constant background noise level of $\operatorname{PSD}_{\mathrm{n}}=10^{-9} \mathrm{nT}^{2} \mathrm{~Hz}^{-1}$ during the entire observational period. The SNR is then derived from

$$
\mathrm{SNR}=\frac{\mathrm{PSD}_{\text {meas }}-\mathrm{PSD}_{\mathrm{n}}}{\mathrm{PSD}_{\mathrm{n}}}
$$


with $\mathrm{PSD}_{\text {meas }}$ as the measured total auto-power spectral density. This yields the black profile shown in Fig. 4a. Moreover, this figure contains a profile for the planarity, drawn in blue color. As can be seen, measured planarities get close to 1 at times when chorus emission is picked up by THEMIS-D. In between, planarity values drop and fluctuate more heavily. The starting period between second $\sim 1.5$ and 2.0 (far left side of Fig. 4) mainly belongs to background noise. We notice that, even for noisy emission, the planarity rarely drops below $\sim 0.5$, which is a consequence of a limited number of points used for spectral averaging ( 7 neighboring windows in time, 7 neighboring frequency channels).

Strong fluctuations for the very same noisy period are also recognizable for other parameters shown in Fig. $4 \mathrm{~b}$, c. The various versions of $D_{\mathrm{p}}$ do not differ much inside chorus elements, i.e., in the presence of a strong and highly polarized signal. All $D_{\mathrm{p}}$ are at levels close to 1 . In between chorus elements and during the noisy starting period, fluctuations are stronger. Without going into too much detail, we can note that differences between $D_{\mathrm{p} 3}$ (green) and $D_{\mathrm{p} 3 e}$ (red) seem to be marginal, and that the profile for $D_{\mathrm{p} 2}$ (blue) usually remains on top ( $D_{\mathrm{p} 2}$ always assumes the presence of a plane wave). Fluctuations are strongest for $D_{\mathrm{p} 3 i}$ (orange). It is interesting that $D_{\mathrm{p} 3 i}$ significantly overestimates the true degree of polarization during the noisy starting period between second $\sim 1.5$ and 2.0 , but it is dropping stronger than the other versions of $D_{\mathrm{p}}$ at times of lowered planarity in between the chorus elements. As can be seen at time $\sim 3.6 \mathrm{~s}, D_{\mathrm{p} 3 i}$ might even become slightly negative. This is explained by an increased $\lambda_{0}$ (see Eq. (75)) originating from a stronger residual polarized component along the wave-frame's $z$-direction.

A similar behavior among all three definitions of ellipticity and the degree of circular polarization can be concluded from Fig. 4c. As expected for the magnetic field of whistlermode chorus, values are very close to $+1(\mathrm{RH})$. During the noisy starting period, fluctuations are a bit stronger for $\varepsilon_{s}$ (green) than $\varepsilon_{\mathrm{p}}$ (red) and $\varepsilon_{2}$ (blue). Later on, all three profiles are almost identical. It seems that even in between the chorus elements THEMIS-D is picking up circularly polarized radiation, which might be weak whistler-mode hiss emission. Nevertheless, measured values for ellipticity must be handled with caution due to low values for the planarity measured for those times.

Finally, wave normal angles are displayed as a black profile in Fig. 4c. Prior to the wave propagation SVD analysis according to Sect. 1.2, THEMIS-D waveforms have been rotated from the antenna reference frame into an orthogonal field-aligned coordinate system. Thus, the angle $\theta_{k}$ is the angle between the wave vector $\mathbf{k}$ and the direction of the ambient planetary magnetic field. Small values $\left(<20^{\circ}\right)$ for $\theta_{k}$ indicate field-aligned propagation for chorus waves. In between and during the noisy starting period, $\theta_{k}$ fluctuates strongly, reaching even $\sim 90^{\circ}$. Anyhow, results for $\theta_{k}$ should only be interpreted for planarities $>0.8$, i.e., when the assumption of the presence of a plane wave is adequately fulfilled.

\section{Summary and Conclusions}

We exploit the hypothesis that any measured signal can be considered as a linear superposition of a fully polarized part and a completely unpolarized part, which we call noise. In connection with the assumption of the presence of a single plane wave, the meaning of singular values of the Hermitian spectral matrix, which are calculated from singular value decomposition (SVD), is investigated.

As it turns out, $\lambda_{1}$ from the complex spectral matrix yields an estimate for the isotropic noise power inside the two-dimensional plane of the wavefront. $\lambda_{1}$ contains portions from 
isotropic background noise and from the unpolarized part of a partially polarized plane wave. The assumption of a plane wave implies that $\lambda_{0}$ contains only the isotropic noise component. Under realistic conditions, i.e., taking into account a violation of the plane wave assumption, $\lambda_{0}$ also contains some fraction of the residual polarized power $\left(J_{\mathrm{p}, z z}\right)$ which cannot be restricted to a plane. Care should be taken that if $J_{\mathrm{p}, z z}$ exceeds the signal's unpolarized power, then $\lambda_{0}>\lambda_{1}$. The general strategy of assigning the smallest singular value to $\lambda_{0}$ and the medium singular value to $\lambda_{1}$ is not valid anymore for a complex spectral matrix. A comparison to singular values computed from the real part of the spectral matrix helps to resolve this issue. For this real matrix, as well as for the $6 \times 3$ spectral matrix from Santolík et al. (2003), the singular value containing the isotropic noise component plus a possible $J_{\mathrm{p}, z z}$ will always be the smallest one.

The singular values computed from SVD of the real part of the spectral matrix always represent a sum of powers from noise and polarized signal along the three main axes of the polarization ellipsoid. They can be used for computing a parameter for the wave's planarity similar to the method of Santolík et al. (2003), and they can be combined with the singular values from complex SVD to get a noise-free estimate for the wave's ellipticity (see Eq. (72)). Unfortunately, the singular values from real SVD do not provide enough additional information for a complete separation of noise and polarized signal if the assumption of a plane wave is violated (planarity $<1$ ). Such a complete separation would be highly desirable for an accurate specification of the degree of polarization. However, we find that the definition from Ellis et al. (2005) for the degree of polarization incorporates all available information one can obtain from the spectral matrix about a separation between noise and the polarized signal from a plane wave. Whereas Ellis et al. (2005) diagonalize the spectral matrix directly and rearrange terms afterward, we choose the opposite approach, by first modeling the spectral matrices for noise and polarized signal and then investigating the results of a diagonalization, i.e., the singular values. This provides additional insight into the problem and clearly illustrates limitations imposed on the system.

The reliability of computed polarization parameters can be estimated depending on a signal-to-noise ratio (SNR) or a planarity parameter. Both depend, in turn, on the parameters chosen for spectral averaging (in time and frequency). Considering a variety of possible weighting functions for averaging (rectangular, Hanning, Hamming, ...) and different numbers of time windows and frequency channels used for averaging, makes it difficult to provide explicit numbers for confidence levels. However, as a rule of thumb, little spectral averaging (over $\sim 7$ time-windows/frequency channels) will require a SNR $\geq 10$ (polarized signal $\geq 10 \mathrm{~dB}$ above noise level) or a planarity $\geq 0.8$ for resolving the correct degree of polarization and ellipticity within a few percent inaccuracy, and for resolving the correct wave normal direction within a $\sim 5^{\circ}$ error margin.

Errors in $\theta_{k}$ as a function of the number $N$ of frequency channels used for averaging are summarized in Fig. 5. These are results from a simulation of a $100 \%$ polarized signal with varying SNR and isotropic white noise. Corresponding waveforms are modeled as already outlined in Sect. 5.2. Over 21 runs, the SNR is modified logarithmically between $10^{-1}$ and $10^{3}$, but the signal's polarization, frequency $\left(f=0.5 f_{N y}\right)$ and wave normal angle $\left(\theta_{k}=20^{\circ}\right)$ are fixed. Each of this series of 21 runs is repeated with 25 different values for the number of samples with which waveforms are generated in the time domain, resulting in a different number $N$ of frequency channels between $0 \leq f \leq f_{N y}$ which are available for averaging (rectangular weighting function), where $N$ is logarithmically increasing from 3 to 1000 . Since waveforms are modeled for only one window in the time domain, no averaging in time is performed here. Finally, the entire process is repeated 500 times, each time with a newly computed random series for white noise. Thus, each bin in Fig. 5 contains an average error in $\theta_{k}$ which is 

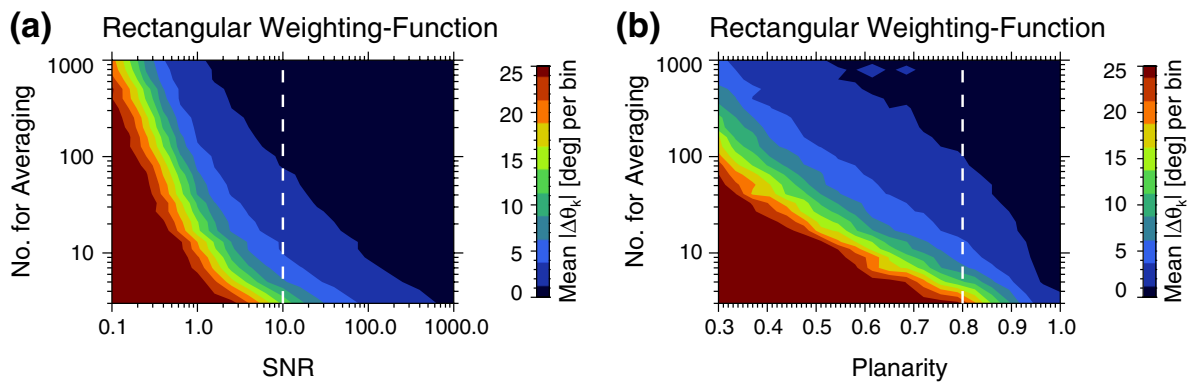

Fig. 5 Absolute value of error in wave normal angle $\left(\left|\Delta \theta_{k}\right|\right)$ as a function of a SNR and $\mathbf{b}$ planarity ( $x$-axis) and the number of frequency channels over which spectral averaging is performed ( $y$-axis)

computed from 500 values. Figure $5 \mathrm{~b}$ is the result of rebinning values with respect to planarity along the $x$-axis.

In general, errors in $\theta_{k}$ can be expected to decrease with a factor $\sqrt{N}$ because the influence of a randomly polarized component inherent in the noise becomes suppressed with the very same factor. Larger $N$ values allow for setting lower limits with respect to SNR and planarity, but this implicates the disadvantage of reduced spectral resolution. For example, uncertainties on the order of $\sim 5^{\circ}$ for $\theta_{k}$ can as well be obtained for SNR $\sim 1$ and for a planarity of 0.5 when we average over $\sim 100$ matrices (see Fig. 5).

In conclusion, we have presented here an overview of methods to analyze the wave polarization in the presence of noise. Newly developed techniques are shown in the context of former development. We hope that these new methods will be useful for analysis of multicomponent data which becomes a standard in the experimental investigation of waves and instabilities in space plasmas.

Acknowledgements THEMIS data used to produce Figs. 3 and 4 as well as processing software can be downloaded from the University of California, Berkeley, at http://themis.ssl.berkeley.edu/data/themis/thd/. For more information on accessibility and handling of the data, please contact the corresponding author at ut@ufa.cas.cz. This work has been supported from the Mobility Plus grant JSPS-17-14 and from the Praemium Academiae Award of the Czech Academy of Sciences. O.S. also acknowledges support from the LTAUSA17070 grant.

Open Access This article is distributed under the terms of the Creative Commons Attribution 4.0 International License (http://creativecommons.org/licenses/by/4.0/), which permits unrestricted use, distribution, and reproduction in any medium, provided you give appropriate credit to the original author(s) and the source, provide a link to the Creative Commons license, and indicate if changes were made.

\section{Appendix A: Manipulation of $D_{p 2}$}

For a $2 \times 2$ spectral matrix $\mathbf{J}$,

$$
\begin{aligned}
\operatorname{tr}(\mathbf{J} \cdot \mathbf{J}) & =J_{x x}^{2}+2 J_{x y} J_{x y}^{*}+J_{y y}^{2} \\
& =J_{x x}^{2}+J_{y y}^{2}+2 J_{x x} J_{y y}-2 J_{x x} J_{y y}+2 J_{x y} J_{x y}^{*} \\
& =\left(J_{x x}+J_{y y}\right)^{2}-2\left(J_{x x} J_{y y}-J_{x y} J_{x y}^{*}\right) \\
& =\operatorname{tr}^{2}(\mathbf{J})-2 \operatorname{det}(\mathbf{J}) .
\end{aligned}
$$


The expression for the 2D degree of polarization using the Stokes parameters gets manipulated according to

$$
\begin{aligned}
D_{\mathrm{p} 2}^{2} & =\frac{Q^{2}+U^{2}+V^{2}}{S^{2}} \\
& =\frac{\left(J_{x x}-J_{y y}\right)^{2}+\left(J_{x y}+J_{x y}^{*}\right)^{2}-\left(J_{x y}^{*}-J_{x y}\right)^{2}}{\left(J_{x x}+J_{y y}\right)^{2}} \\
& =\frac{J_{x x}^{2}-2 J_{x x} J_{y y}+J_{y y}^{2}+J_{x y}^{2}+2 J_{x y} J_{x y}^{*}+\left(J_{x y}^{*}\right)^{2}-\left(J_{x y}^{*}\right)^{2}+2 J_{x y} J_{x y}^{*}-J_{x y}^{2}}{\operatorname{tr}^{2}(\mathbf{J})} \\
& =\frac{\left(J_{x x}^{2}+J_{y y}^{2}+2 J_{x y} J_{x y}^{*}\right)-2\left(J_{x x} J_{y y}-J_{x y} J_{x y}^{*}\right)}{\operatorname{tr}^{2}(\mathbf{J})} \\
& =\frac{\operatorname{tr}^{2}\left(\mathbf{J}^{2}\right)-2 \operatorname{det}(\mathbf{J})}{\operatorname{tr}^{2}(\mathbf{J})} \\
D_{\mathrm{p} 2}^{2} & =\frac{2 \operatorname{tr}^{2}\left(\mathbf{J}^{2}\right)}{\operatorname{tr}^{2}(\mathbf{J})}-1, \text { or }=1-\frac{4 \operatorname{det}(\mathbf{J})}{\operatorname{tr}^{2}(\mathbf{J})} .
\end{aligned}
$$

Thus, the $2 \mathrm{D}$ degree of polarization can be formulated in terms of the invariants of the spectral matrix, i.e., in terms of $\operatorname{det}(\mathbf{J}), \operatorname{tr}(\mathbf{J})$ and $\operatorname{tr}\left(\mathbf{J}^{2}\right)$.

\section{Appendix B: Factor $K$ for $D_{p 3}$}

In

$$
D_{\mathrm{p} 3}=K \frac{\left(\sigma_{1}^{2}+\sigma_{2}^{2}+\cdots+\sigma_{8}^{2}\right)^{\frac{1}{2}}}{\sigma_{0}},
$$

the factor $K$ shall be adjusted so that $D_{\mathrm{p} 3}=1$ for a fully polarized wave. If we assume a plane wave with its oscillatory components confined to the $[x, y]$-plane, then all terms in the $3 \mathrm{D}$ Stokes parameters which include a $z$-component are zero. Furthermore, the determinant of the $2 \times 2$ sub-matrix $\mathbf{J}_{\mathrm{p}}$ in the upper left-hand corner of $\mathbf{J}$ is zero, i.e., $J_{x x} J_{y y}=J_{x y} J_{x y}^{*}$ (see also "Appendix E"). Inserting the 3D Stokes parameters into the definition for the 3D degree of polarization yields

$$
\begin{aligned}
D_{\mathrm{p} 3}^{2}= & K^{2} \frac{9}{4} \frac{J_{x y}^{2}+2 J_{x y} J_{x y}^{*}+\left(J_{x y}^{*}\right)^{2}-J_{x y}^{2}+2 J_{x y} J_{x y}^{*}-\left(J_{x y}^{*}\right)^{2}+J_{x x}^{2}-2 J_{x x} J_{y y}+J_{y y}^{2}}{\left(J_{x x}+J_{y y}\right)^{2}} \\
& +1 / 3\left(J_{x x}+J_{y y}\right)^{2} \\
= & K^{2} \frac{9}{4} \frac{4 J_{x y} J_{x y}^{*}+J_{x x}^{2}-2 J_{x x} J_{y y}+J_{y y}^{2}+1 / 3\left(J_{x x}^{2}+2 J_{x x} J_{y y}+J_{y y}^{2}\right)}{\left(J_{x x}+J_{y y}\right)^{2}} \\
= & 3 K^{2} \frac{3 J_{x y} J_{x y}^{*}+J_{x x}^{2}-J_{x x} J_{y y}+J_{y y}^{2}}{\left(J_{x x}+J_{y y}\right)^{2}} \\
= & 3 K^{2} \frac{J_{x x}^{2}+2 J_{x x} J_{y y}+J_{y y}^{2}}{\left(J_{x x}+J_{y y}\right)^{2}} \\
= & 3 K^{2} \frac{\left(J_{x x}+J_{y y}\right)^{2}}{\left(J_{x x}+J_{y y}\right)^{2}} \\
= & 3 K^{2}
\end{aligned}
$$


Thus, the normalization factor $\mathrm{K}$ must be $1 / \sqrt{3}$.

\section{Appendix C: Diagonalization of J According to Ellis et al. (2005)}

$$
\begin{aligned}
\mathbf{J} & =\mathbf{U} \cdot \boldsymbol{\Lambda} \cdot \mathbf{U}^{H} \\
& =\lambda_{2} \mathbf{U} \cdot\left(\begin{array}{lll}
1 & 0 & 0 \\
0 & 0 & 0 \\
0 & 0 & 0
\end{array}\right) \cdot \mathbf{U}^{H}+\lambda_{1} \mathbf{U} \cdot\left(\begin{array}{lll}
0 & 0 & 0 \\
0 & 1 & 0 \\
0 & 0 & 0
\end{array}\right) \cdot \mathbf{U}^{H}+\lambda_{0} \mathbf{U} \cdot\left(\begin{array}{lll}
0 & 0 & 0 \\
0 & 0 & 0 \\
0 & 0 & 1
\end{array}\right) \cdot \mathbf{U}^{H} \\
& =\lambda_{2} \boldsymbol{\Gamma}_{\mathbf{2}}+\lambda_{1} \boldsymbol{\Gamma}_{\mathbf{1}}+\lambda_{0} \boldsymbol{\Gamma}_{\mathbf{0}},
\end{aligned}
$$

with

$$
\boldsymbol{\Gamma}_{2}=\mathbf{U} \cdot\left(\begin{array}{lll}
1 & 0 & 0 \\
0 & 0 & 0 \\
0 & 0 & 0
\end{array}\right) \cdot \mathbf{U}^{H}, \quad \boldsymbol{\Gamma}_{1}=\mathbf{U} \cdot\left(\begin{array}{lll}
0 & 0 & 0 \\
0 & 1 & 0 \\
0 & 0 & 0
\end{array}\right) \cdot \mathbf{U}^{H}, \quad \boldsymbol{\Gamma}_{0}=\mathbf{U} \cdot\left(\begin{array}{ccc}
0 & 0 & 0 \\
0 & 0 & 0 \\
0 & 0 & 1
\end{array}\right) \cdot \mathbf{U}^{H}
$$

Now,

$$
\begin{aligned}
\mathbf{J} & =\lambda_{2} \boldsymbol{\Gamma}_{\mathbf{2}}+\lambda_{1}\left(\boldsymbol{\Gamma}_{\mathbf{1}}+\boldsymbol{\Gamma}_{\mathbf{2}}\right)-\lambda_{1} \boldsymbol{\Gamma}_{\mathbf{2}}+\lambda_{0}\left(\boldsymbol{\Gamma}_{\mathbf{2}}+\boldsymbol{\Gamma}_{\mathbf{1}}+\boldsymbol{\Gamma}_{\mathbf{0}}\right)-\lambda_{0} \boldsymbol{\Gamma}_{\mathbf{2}}-\lambda_{0} \boldsymbol{\Gamma}_{\mathbf{1}} \\
& =\left(\lambda_{2}-\lambda_{1}\right) \boldsymbol{\Gamma}_{\mathbf{2}}+\left(\lambda_{1}-\lambda_{0}\right)\left(\boldsymbol{\Gamma}_{\mathbf{2}}+\boldsymbol{\Gamma}_{\mathbf{1}}\right)+\lambda_{0}\left(\boldsymbol{\Gamma}_{\mathbf{2}}+\boldsymbol{\Gamma}_{\mathbf{1}}+\boldsymbol{\Gamma}_{\mathbf{0}}\right) \\
& =\left(\lambda_{2}-\lambda_{1}\right) \mathbf{U} \cdot\left(\begin{array}{lll}
1 & 0 & 0 \\
0 & 0 & 0 \\
0 & 0 & 0
\end{array}\right) \cdot \mathbf{U}^{H}+\left(\lambda_{1}-\lambda_{0}\right) \mathbf{U} \cdot\left(\begin{array}{lll}
1 & 0 & 0 \\
0 & 1 & 0 \\
0 & 0 & 0
\end{array}\right) \cdot \mathbf{U}^{H}+\lambda_{0} \mathbf{U} \cdot\left(\begin{array}{lll}
1 & 0 & 0 \\
0 & 1 & 0 \\
0 & 0 & 1
\end{array}\right) \cdot \mathbf{U}^{H} .
\end{aligned}
$$

\section{Appendix D: Singular Values and Eigenvalues of a Hermitian Matrix}

Here we prove that for a Hermitian spectral matrix, a singular value and singular vector is the same as an eigenvalue and an eigenvector, respectively.

The eigenvalue problem is:

$$
\begin{aligned}
& \mathbf{J} \cdot \mathbf{u}_{i}=\lambda_{i} \mathbf{u}_{i}, \\
& \mathbf{J} \cdot \mathbf{v}_{i}=\lambda_{i} \mathbf{v}_{i} .
\end{aligned}
$$

Manipulating Eq. (80) yields

$$
\begin{aligned}
\mathbf{J} \cdot \mathbf{u}_{i} & =\lambda_{i} \mathbf{u}_{i} \quad \mid \cdot \lambda_{i} \\
\mathbf{J} \cdot\left(\lambda_{i} \mathbf{u}_{i}\right) & =\lambda_{i}^{2} \mathbf{u}_{i} \quad \mid \text { re- insert equation (82) on left- hand side again } \\
\mathbf{J} \cdot \mathbf{J} \cdot \mathbf{u}_{i} & =\lambda_{i}^{2} \mathbf{u}_{i} \quad \mid \text { use } \mathbf{J} \cdot \mathbf{J}=\mathbf{J} \cdot \mathbf{J}^{H} \\
\mathbf{J} \cdot \mathbf{J}^{H} \cdot \mathbf{u}_{i} & =\lambda_{i}^{2} \mathbf{u}_{i}
\end{aligned}
$$

Manipulating Eq. (81) analogously, and using $\mathbf{J} \cdot \mathbf{J}=\mathbf{J}^{H} \cdot \mathbf{J}$, yields 


$$
\begin{gathered}
\mathbf{J} \cdot \mathbf{v}_{i}=\lambda_{i} \mathbf{v}_{i} \quad \mid \cdot \lambda_{i} \\
\cdot \\
\cdot \\
\mathbf{J}^{H} \cdot \mathbf{J} \cdot \mathbf{v}_{i}=\lambda_{i}^{2} \mathbf{v}_{i}
\end{gathered}
$$

The reformulated eigenvalue problem is

$$
\begin{aligned}
& \mathbf{J} \cdot \mathbf{J}^{H} \cdot \mathbf{u}_{i}=\lambda_{i}^{2} \mathbf{u}_{i}, \\
& \mathbf{J}^{H} \cdot \mathbf{J} \cdot \mathbf{v}_{i}=\lambda_{i}^{2} \mathbf{v}_{i} .
\end{aligned}
$$

The singular value problem is:

$$
\begin{gathered}
\mathbf{J}^{H} \cdot \mathbf{u}_{i}=\lambda_{i} \mathbf{v}_{i}, \\
\mathbf{J} \cdot \mathbf{v}_{i}=\lambda_{i} \mathbf{u}_{i} .
\end{gathered}
$$

Applying $\mathbf{J} \cdot$ in Eq. (85) and inserting for the new term $\mathbf{J} \cdot \mathbf{v}_{i}$ the right-hand side of Eq. (86) yields $\mathbf{J} \cdot \mathbf{J}^{H} \cdot \mathbf{u}_{i}=\lambda_{i}^{2} \mathbf{u}_{i}$. Applying $\mathbf{J}^{H} \cdot$ in Eq. (86) and inserting for the new term $\mathbf{J}^{H} \cdot \mathbf{u}_{i}$ the right-hand side of Eq. (85) yields $\mathbf{J}^{H} \cdot \mathbf{J} \cdot \mathbf{v}_{i}=\lambda_{i}^{2} \mathbf{v}_{i}$.

The reformulated singular value problem is

$$
\begin{aligned}
& \mathbf{J} \cdot \mathbf{J}^{H} \cdot \mathbf{u}_{i}=\lambda_{i}^{2} \mathbf{u}_{i}, \\
& \mathbf{J}^{H} \cdot \mathbf{J} \cdot \mathbf{v}_{i}=\lambda_{i}^{2} \mathbf{v}_{i} .
\end{aligned}
$$

Since $(87)=(83)$ and $(88)=(84)$, the singular value problem is the same as the eigenvalue problem for a Hermitian spectral matrix.

\section{Appendix E: Proof of $\operatorname{det}\left(J_{p}\right)=0$}

In three dimensions, the determinant of the $3 \times 3$ matrix

$$
\mathbf{J}_{\mathrm{p}}=\left(\begin{array}{ccc}
J_{x x} & J_{x y} & 0 \\
J_{x y}^{*} & J_{y y} & 0 \\
0 & 0 & 0
\end{array}\right)
$$

will always be zero. In two dimensions, the determinant of the $2 \times 2$ sub-matrix in the upper left-hand corner is

$$
\begin{aligned}
\operatorname{det}\left(\mathbf{J}_{\mathrm{p}}\right) & =J_{x x} J_{y y}-J_{x y} J_{x y}^{*} \\
& =\left\langle B_{x} B_{x}^{*}\right\rangle\left\langle B_{y} B_{y}^{*}\right\rangle-\left\langle B_{x} B_{y}^{*}\right\rangle\left\langle B_{y} B_{x}^{*}\right\rangle .
\end{aligned}
$$

For a fully polarized signal, the averaging operator $\langle\ldots\rangle$ over the correlation products can be omitted. The first term on the right-hand side of Eq. (89) is

$$
\begin{aligned}
B_{x} B_{x}^{*} & =\left[\Re\left\{B_{x}\right\}+i \mathfrak{I}\left\{B_{x}\right\}\right]\left[\mathfrak{R}\left\{B_{x}\right\}-i \mathfrak{I}\left\{B_{x}\right\}\right] \\
& =\mathfrak{R}^{2}\left\{B_{x}\right\}+\mathfrak{J}^{2}\left\{B_{x}\right\} .
\end{aligned}
$$


The first part of the sum becomes

$$
\left(B_{x} B_{x}^{*}\right)\left(B_{y} B_{y}^{*}\right)=\mathfrak{R}^{2}\left\{B_{x}\right\} \mathfrak{R}^{2}\left\{B_{y}\right\}+\mathfrak{R}^{2}\left\{B_{y}\right\} \mathfrak{\Im}^{2}\left\{B_{x}\right\}+\mathfrak{R}^{2}\left\{B_{x}\right\} \mathfrak{\Im}^{2}\left\{B_{y}\right\}+\mathfrak{J}^{2}\left\{B_{x}\right\} \mathfrak{\Im}^{2}\left\{B_{y}\right\} .
$$

The third term on the right-hand side of Eq. (89) is

$$
\begin{aligned}
B_{x} B_{y}^{*} & =\left[\mathfrak{R}\left\{B_{x}\right\}+i \mathfrak{I}\left\{B_{x}\right\}\right]\left[\mathfrak{R}\left\{B_{y}\right\}-i \mathfrak{I}\left\{B_{y}\right\}\right] \\
& =\mathfrak{R}\left\{B_{x}\right\} \mathfrak{R}\left\{B_{y}\right\}+\mathfrak{I}\left\{B_{x}\right\} \mathfrak{J}\left\{B_{y}\right\}+i\left[\mathfrak{R}\left\{B_{y}\right\} \mathfrak{I}\left\{B_{x}\right\}-\mathfrak{R}\left\{B_{x}\right\} \mathfrak{I}\left\{B_{y}\right\}\right] .
\end{aligned}
$$

The second part of the sum becomes

$$
\begin{aligned}
\left(B_{x} B_{y}^{*}\right)\left(B_{y} B_{x}^{*}\right) & =\left(B_{x} B_{y}^{*}\right)\left(B_{x} B_{y}^{*}\right)^{*} \\
& =\left[\mathfrak{R}\left\{B_{x}\right\} \mathfrak{R}\left\{B_{y}\right\}+\mathfrak{I}\left\{B_{x}\right\} \mathfrak{I}\left\{B_{y}\right\}\right]^{2}+\left[\mathfrak{R}\left\{B_{y}\right\} \mathfrak{I}\left\{B_{x}\right\}-\mathfrak{R}\left\{B_{x}\right\} \mathfrak{\Im}\left\{B_{y}\right\}\right]^{2} \\
& =\mathfrak{R}^{2}\left\{B_{x}\right\} \mathfrak{R}^{2}\left\{B_{y}\right\}+\mathfrak{J}^{2}\left\{B_{x}\right\} \mathfrak{J}^{2}\left\{B_{y}\right\}+\mathfrak{R}^{2}\left\{B_{y}\right\} \mathfrak{J}^{2}\left\{B_{x}\right\}+\mathfrak{R}^{2}\left\{B_{x}\right\} \mathfrak{J}^{2}\left\{B_{y}\right\} .
\end{aligned}
$$

Substituting both parts into Eq. (89) yields

$$
\operatorname{det}\left(\mathbf{J}_{\mathrm{p}}\right)=0 \text {. }
$$

\section{References}

Angelopoulos V (2008) The THEMIS mission. Space Sci Rev 141:5-34. https://doi.org/10.1007/s1121 4-008-9336-1

Barakat R (1977) Degree of polarization and the principal idempotents of the coherency matrix. Opt Commun 23:147-150. https://doi.org/10.1016/0030-4018(77)90292-9

Berthelier JJ, Godefroy M, Leblanc F, Malingre M, Menvielle M, Lagoutte D, Brochot JY, Colin F, Elie F, Legendre C, Zamora P, Benoist D, Chapuis Y, Artru J, Pfaff R (2006) ICE, the electric field experiment on DEMETER. Planet Space Sci 54:456-471. https://doi.org/10.1016/j.pss.2005.10.016

Bonnell JW, Mozer FS, Delory GT, Hull AJ, Ergun RE, Cully CM, Angelopoulos V, Harvey PR (2008) The electric field instrument (EFI) for THEMIS. Space Sci Rev 141:303-341. https://doi.org/10.1007/ s11214-008-9469-2

Born M, Wolf E (1999) Principles of optics, 7th edn. Cambridge University Press, Cambridge

Chen L, Santolík O, Hajoš M, Zheng L, Zhima Z, Heelis R, Hanzelka M, Horne RB, Parrot M (2017) Source of the low-altitude hiss in the ionosphere. Geophys Res Lett 44:2060-2069. https://doi. org/10.1002/2016GL072181

Cornilleau-Wehrlin N, Chauveau P, Louis S, Meyer A, Nappa JM, Perraut S, Rezeau L, Robert P, Roux A, de Villedary C, de Conchy Y, Friel L, Harvey CC, Hubert D, Lacombe C, Manning R, Wouters F, Lefeuvre F, Parrot M, Pincon JL, Poirier B, Kofman W, Louarn P (1997) The cluster spatiotemporal analysis of field fluctuations (STAFF) experiment. Space Sci Rev 79:107-136. https://doi. org/10.1023/A:1004979209565

Demekhov AG, Taubenschuss U, Santolík O (2017) Simulation of VLF chorus emissions in the magnetosphere and comparison with THEMIS spacecraft data. J Geophys Res (Space Phys) 122:166-184. https://doi.org/10.1002/2016JA023057

Dennis MR (2004) Geometric interpretation of the three-dimensional coherence matrix for nonparaxial polarization. J Opt A Pure Appl Opt 6:S26-S31. https://doi.org/10.1088/1464-4258/6/3/005

Ellis J, Dogariu A (2005) On the degree of polarization of random electromagnetic fields. Opt Commun 253:257-265. https://doi.org/10.1016/j.optcom.2005.05.020

Ellis J, Dogariu A, Ponomarenko S, Wolf E (2005) Degree of polarization of statistically stationary electromagnetic fields. Opt Commun 248:333-337. https://doi.org/10.1016/j.optcom.2004.12.050

Fano U (1957) Description of states in quantum mechanics by density matrix and operator techniques. Rev Modern Phys 29:74-93. https://doi.org/10.1103/RevModPhys.29.74

Fischer G, Cecconi B, Lamy L, Ye SY, Taubenschuss U, Macher W, Zarka P, Kurth WS, Gurnett DA (2009) Elliptical polarization of Saturn Kilometric Radiation observed from high latitudes. J Geophys Res (Space Phys) 114:A08216. https://doi.org/10.1029/2009JA014176 
Fowler RA, Kotick BJ, Elliott RD (1967) Polarization analysis of natural and artificially induced geomagnetic micropulsations. J Geophys Res 72:2871-2883. https://doi.org/10.1029/JZ072i011p02871

Gell-Mann M (1962) Symmetries of baryons and mesons. Phys Rev 125:1067-1084. https://doi. org/10.1103/PhysRev.125.1067

Gil JJ (2007) Polarimetric characterization of light and media. Physical quantities involved in polarimetric phenomena. Eur Phys J Appl Phys 40:1-47. https://doi.org/10.1051/epjap:2007153

Gil JJ (2014) Interpretation of the coherency matrix for three-dimensional polarization states. Phys Rev 90(4):043858. https://doi.org/10.1103/PhysRevA.90.043858

Gil JJ, San José I (2010) 3D polarimetric purity. Opt Commun 283:4430-4434. https://doi.org/10.1016/j. optcom.2010.04.090

Gurnett DA (1974) The earth as a radio source: terrestrial kilometric radiation. J Geophys Res 79:42274238. https://doi.org/10.1029/JA079i028p04227

Gurnett DA, Persoon AM, Randall RF, Odem DL, Remington SL, Averkamp TF, Debower MM, Hospodarsky GB, Huff RL, Kirchner DL, Mitchell MA, Pham BT, Phillips JR, Schintler WJ, Sheyko P, Tomash DR (1995) The polar plasma wave instrument. Space Sci Rev 71:597-622. https://doi. org/10.1007/BF00751343

Gurnett DA, Kurth WS, Kirchner DL, Hospodarsky GB, Averkamp TF, Zarka P, Lecacheux A, Manning R, Roux A, Canu P, Cornilleau-Wehrlin N, Galopeau P, Meyer A, Boström R, Gustafsson G, Wahlund JE, Åhlen L, Rucker HO, Ladreiter HP, Macher W, Woolliscroft LJC, Alleyne H, Kaiser ML, Desch MD, Farrell WM, Harvey CC, Louarn P, Kellogg PJ, Goetz K, Pedersen A (2004) The Cassini radio and plasma wave investigation. Space Sci Rev 114:395-463. https://doi.org/10.1007/ s11214-004-1434-0

Hanzelka M, Santolík O, Hajoš M, Němec F, Parrot M (2017) Observation of ionospherically reflected quasiperiodic emissions by the DEMETER spacecraft. Geophys Res Lett 44:8721-8729. https:// doi.org/10.1002/2017GL074883

Hartley DP, Kletzing CA, Kurth WS, Hospodarsky GB, Bounds SR, Averkamp TF, Bonnell JW, Santolík O, Wygant JR (2017) An improved sheath impedance model for the Van Allen Probes EFW instrument: effects of the spin axis antenna. J Geophys Res (Space Phys) 122:4420-4429. https://doi. org/10.1002/2016JA023597

IEEE (1998) Standard definitions of terms for radio wave propagation. Institute of Electrical and Electronics Engineers Std. 211-1997. https://doi.org/10.1109/IEEESTD.1998.87897

Kletzing CA, Kurth WS, Acuna M, MacDowall RJ, Torbert RB, Averkamp T, Bodet D, Bounds SR, Chutter M, Connerney J, Crawford D, Dolan JS, Dvorsky R, Hospodarsky GB, Howard J, Jordanova V, Johnson RA, Kirchner DL, Mokrzycki B, Needell G, Odom J, Mark D, Pfaff R, Phillips JR, Piker CW, Remington SL, Rowland D, Santolik O, Schnurr R, Sheppard D, Smith CW, Thorne RM, Tyler J (2013) The electric and magnetic field instrument suite and integrated science (EMFISIS) on RBSP. Space Sci Rev 179:127-181. https://doi.org/10.1007/s11214-013-9993-6

Kurth WS, Imai M, Hospodarsky GB, Gurnett DA, Louarn P, Valek P, Allegrini F, Connerney JEP, Mauk BH, Bolton SJ, Levin SM, Adriani A, Bagenal F, Gladstone GR, McComas DJ, Zarka P (2017) A new view of Jupiter's auroral radio spectrum. Geophys Res Lett 44:7114-7121. https:// doi.org/10.1002/2017GL072889

Le Contel O, Roux A, Robert P, Coillot C, Bouabdellah A, de La Porte B, Alison D, Ruocco S, Angelopoulos V, Bromund K, Chaston CC, Cully C, Auster HU, Glassmeier KH, Baumjohann W, Carlson CW, McFadden JP, Larson D (2008) First results of the THEMIS search coil magnetometers. Space Sci Rev 141:509-534. https://doi.org/10.1007/s11214-008-9371-y

Li W, Ma Q, Thorne RM, Bortnik J, Kletzing CA, Kurth WS, Hospodarsky GB, Nishimura Y (2015) Statistical properties of plasmaspheric hiss derived from Van Allen Probes data and their effects on radiation belt electron dynamics. J Geophys Res (Space Phys) 120:3393-3405. https://doi. org/10.1002/2015JA021048

McPherron RL, Russell CT, Coleman PJ Jr (1972) Fluctuating magnetic fields in the magnetosphere. II: ULF waves. Space Sci Rev 13:411-454. https://doi.org/10.1007/BF00219165

Means JD (1972) Use of the three-dimensional covariance matrix in analyzing the polarization properties of plane waves. J Geophys Res 77:5551-5559. https://doi.org/10.1029/JA077i028p05551

Meredith NP, Thorne RM, Horne RB, Summers D, Fraser BJ, Anderson RR (2003) Statistical analysis of relativistic electron energies for cyclotron resonance with EMIC waves observed on CRRES. J Geophys Res (Space Phys) 108:1250. https://doi.org/10.1029/2002JA009700

Parrot M, Benoist D, Berthelier JJ, Błęcki J, Chapuis Y, Colin F, Elie F, Fergeau P, Lagoutte D, Lefeuvre F, Legendre C, Lévêque M, Pinçon JL, Poirier B, Seran HC, Zamora P (2006) The magnetic field experiment IMSC and its data processing onboard DEMETER: scientific objectives, description and first results. Planet Space Sci 54:441-455. https://doi.org/10.1016/j.pss.2005.10.015 
Píša D, Sulaiman AH, Santolík O, Hospodarsky GB, Kurth WS, Gurnett DA (2018) First observation of Lion roar emission in Saturn's magnetosheath. Geophys Res Lett 45:486-492. https://doi. org/10.1002/2017GL075919

Press WH, Teukolsky SA, Vetterling WT, Flannery BP (1992) Numerical recipes in C. The art of scientific computing. Cambridge University Press, Cambridge

Ripoll JF, Santolík O, Reeves GD, Kurth WS, Denton MH, Loridan V, Thaller SA, Kletzing CA, Turner DL (2017) Effects of whistler mode hiss waves in March 2013. J Geophys Res (Space Phys) 122:7433-7462. https://doi.org/10.1002/2017JA024139

Samson JC (1973) Descriptions of the polarization states of vector processes: applications to ULF magnetic fields. Geophys J 34:403-419. https://doi.org/10.1111/j.1365-246X.1973.tb02404.X

Samson JC, Olson JV (1980) Some comments on the descriptions of the polarization states of waves. Geophys J 61:115-129. https://doi.org/10.1111/j.1365-246X.1980.tb04308.x

Santolík O (2008) New results of investigations of whistler-mode chorus emissions. Nonlinear Process Geophys 15:621-630

Santolík O, Gurnett DA (2002) Propagation of auroral hiss at high altitudes. Geophys Res Lett 29:1481. https://doi.org/10.1029/2001GL013666

Santolík O, Pickett JS, Gurnett DA, Storey LRO (2002) Magnetic component of narrowband ion cyclotron waves in the auroral zone. J Geophys Res (Space Phys) 107:1444. https://doi.org/10.1029/2001JA0001 46

Santolík O, Parrot M, Lefeuvre F (2003) Singular value decomposition methods for wave propagation analysis. Radio Sci 38:1010. https://doi.org/10.1029/2000RS002523

Setälä T, Shevchenko A, Kaivola M, Friberg AT (2002) Degree of polarization for optical near fields. Phys Rev 66(1):016615. https://doi.org/10.1103/PhysRevE.66.016615

Sheppard CJR (2011) Partial polarization in three dimensions. J Opt Soc Am A 28:2655. https://doi. org/10.1364/JOSAA.28.002655

Stokes GG (1852) Über die Veränderung der Brechbarkeit des Lichts. Ann Phys 163:480-490. https://doi. org/10.1002/andp.18521631109

Storey LRO (1953) An investigation of whistling atmospherics. Philos Trans R Soc Lond Ser A 246:113141. https://doi.org/10.1098/rsta.1953.0011

Thompson AR, Moran J, Swenson GW Jr (2017) Interferometry and synthesis in radio astronomy, 3rd edn. Astronomy and Astrophysics Library

Torbert RB, Russell CT, Magnes W, Ergun RE, Lindqvist PA, Le Contel O, Vaith H, Macri J, Myers S, Rau D, Needell J, King B, Granoff M, Chutter M, Dors I, Olsson G, Khotyaintsev YV, Eriksson A, Kletzing CA, Bounds S, Anderson B, Baumjohann W, Steller M, Bromund K, Le G, Nakamura R, Strangeway RJ, Leinweber HK, Tucker S, Westfall J, Fischer D, Plaschke F, Porter J, Lappalainen K (2016) The FIELDS instrument suite on MMS: scientific objectives, measurements, and data products. Space Sci Rev 199:105-135. https://doi.org/10.1007/s11214-014-0109-8

Welch PD (1967) The use of fast Fourier transform for the estimation of power spectra. IEEE Trans Audio Electroacoust 15:70-73. https://doi.org/10.1109/TAU.1967.1161901

Wiener N (1930) Generalized harmonic analysis. Acta Math 55:117-258

Wu CS, Lee LC (1979) A theory of the terrestrial kilometric radiation. Astrophys J 230:621-626. https:// doi.org/10.1086/157120

Zarka P (1998) Auroral radio emissions at the outer planets: observations and theories. J Geophys Res 103:20159-20194. https://doi.org/10.1029/98JE01323 